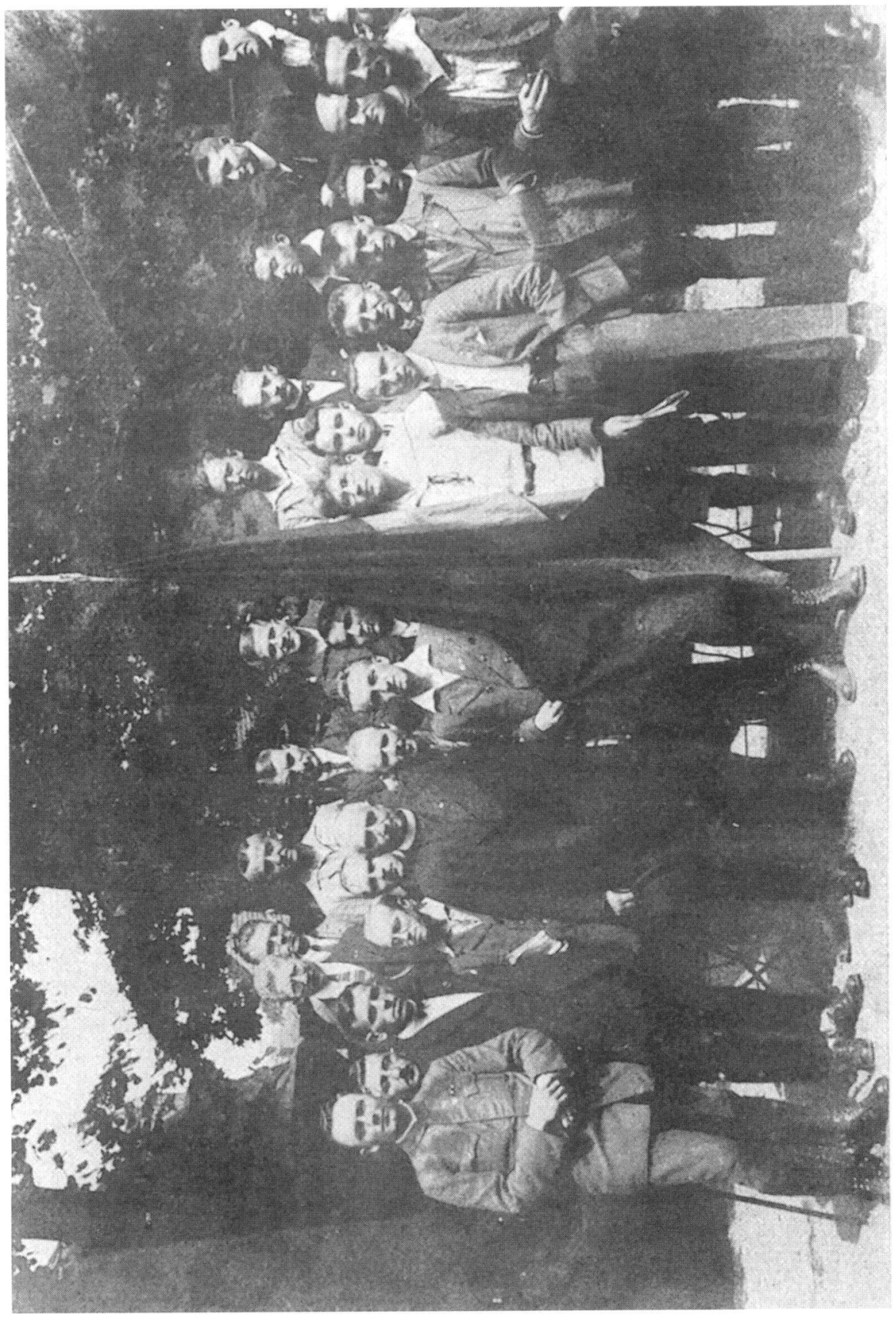

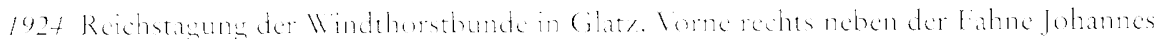
schautt. 


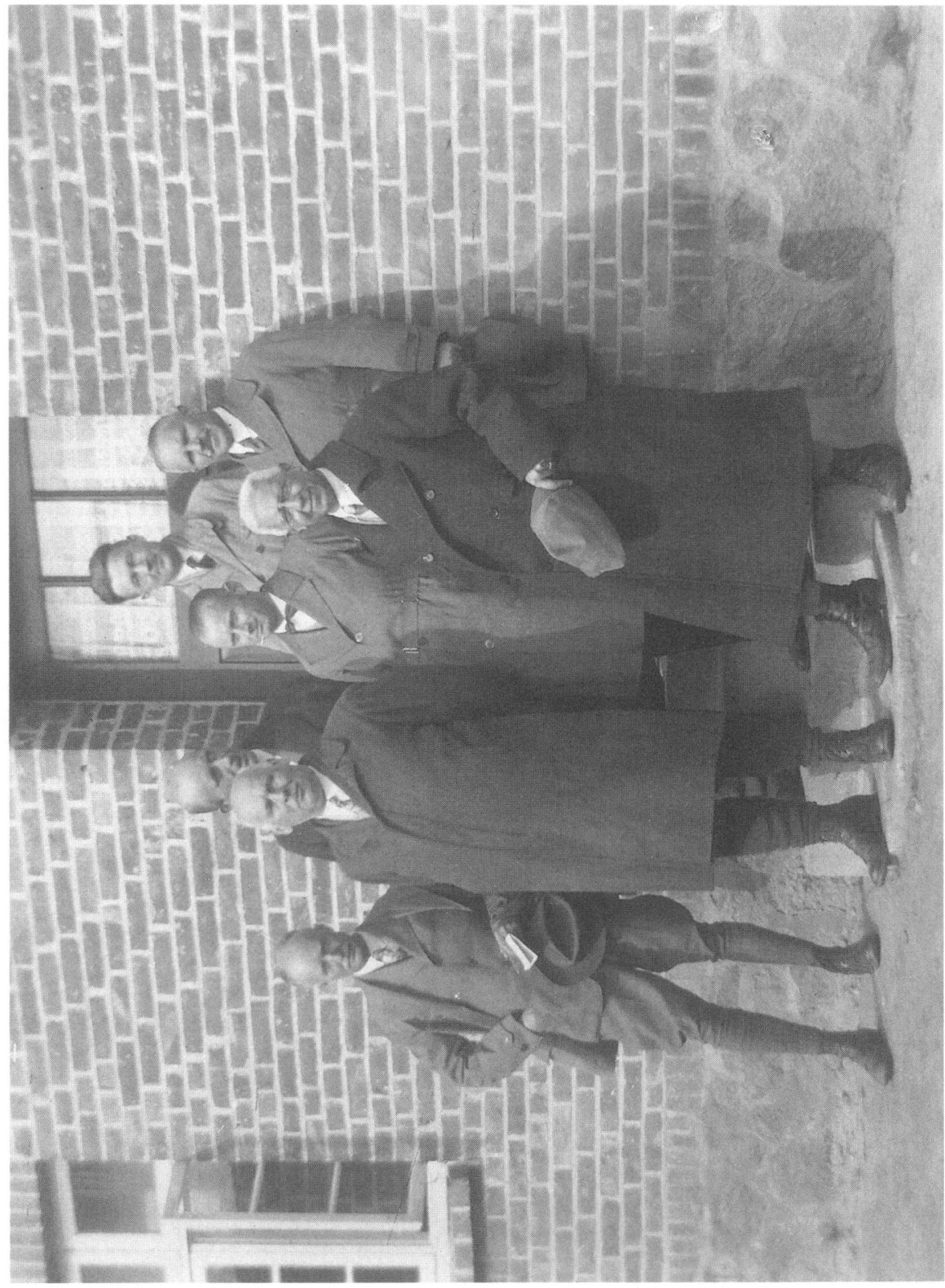

1928 Besichtigungsfahrt einer Delegation der Gesellschaft zur Ï̈rderung der inneren Kolonisation am 1. Oktober 1928 in Mecklenburg. Johannes Sch.uff, in let»ter, erhöhter Position. 


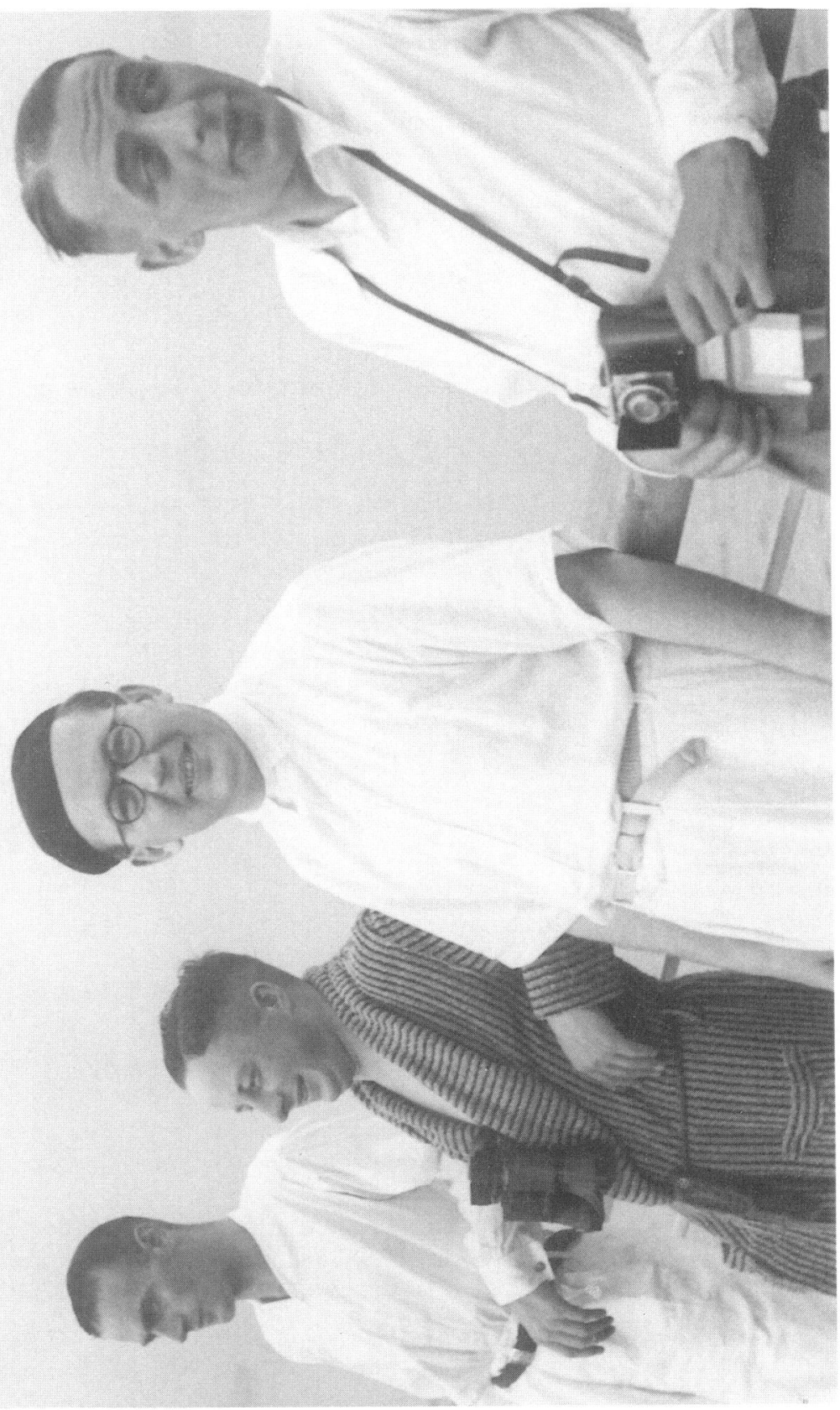

1936 Reise im Auftrag des Völkerbundes zur Erkundung von Siedlungs- bzw. Migrationsmöglichkeiten außerhalb Europas. Johannes Schauff zweiter von rechts, mit Baskenmütze. 


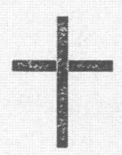

Dr. Johanned Schauif, antigo membro do Parlamento Alemão, Caviuna-Norte do Paraná (Rio de Janeiro-Mosteiro de São Bento)

convida o prezado Snr. e Exma. Familia para uma

\section{Missa Comemorativa}

em surrágio das almas de amigos pessoais e politicos que debaixo da sangrenta perseguição nazista morreram pelas duas conviç̧ơes cristãd :

Erich Klausener, Presidente da Ação Católica - Berlim, assassinado em $3 \sigma$ de Junho de 1934.

Addalbert Probst, Chete da Aśociaçâo de Moçod Católicos, aśsassinado em 30 de Junho de 1934.

Engelbert Dollfuss, Chanceler da Austria, assassinado em 25 de Julbo de 1935.

Farl Schmittmanno, Duesseldort, Professor de Sociologia da Universidade de Colonia,

assausinado em Agosto de 1939.

Albert Hackelsberger, membro do Parlamento oflemâo, morto na pridão em Friburgo em 1940.

Eugen Bolz, Chete do Governo do Estado de 20uerttemberg, executado em 20 de Julho de 1944.

Joseph Wirmer, Advogado, Chete dos Estudanted Catolicod-Berlim, enforcado em 20 de Julho de 1944.

Bernhard Retterhaus, Colonia, Secretario Geral das Associações de Operários Católicos, executado em 20 de Julho de 1944.

Franz Conde de Galen, Merfeld (20estfália), membro da Dieta da Prussia, preso em Julho de 1944 e dedaparecido desde Outubro de 1944

O Requiem será celebrado na Jgreja Abacial de São Bento desta Capital, no dia 21 de Agosto, as 9 boras.

1945 Einladung zu dem von Johannes Schauff initiierten Requiem in Rio de Janeiro zur Erinnerung an die von den Nationalsozialisten ermordeten christlichen deutschen und österreichischen Politikern. 


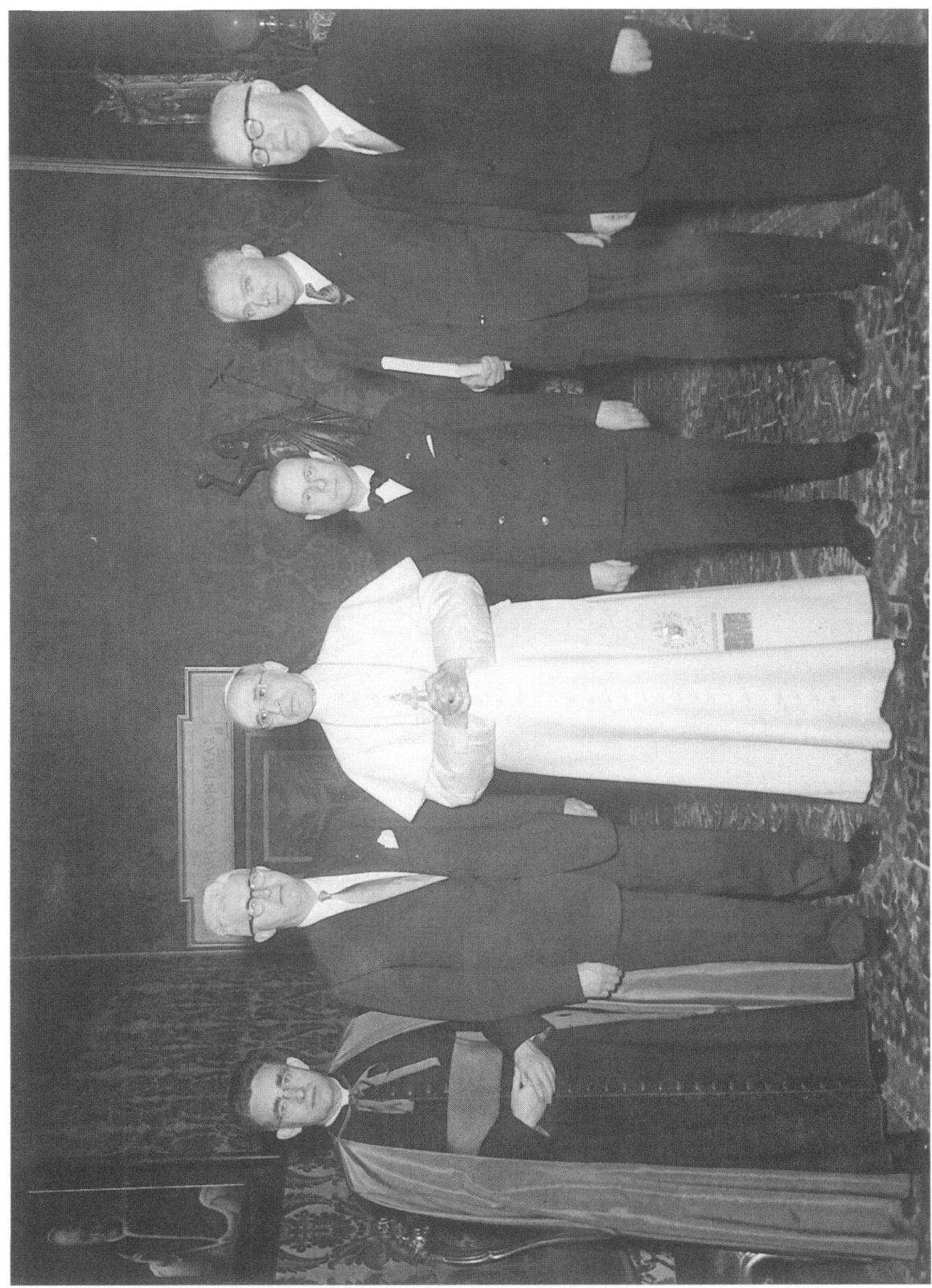

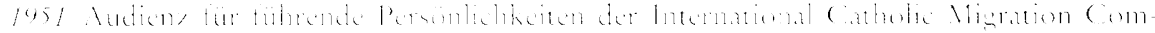

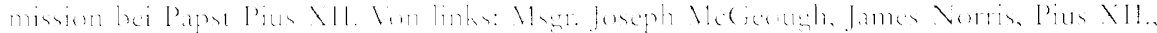

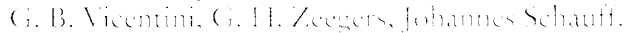




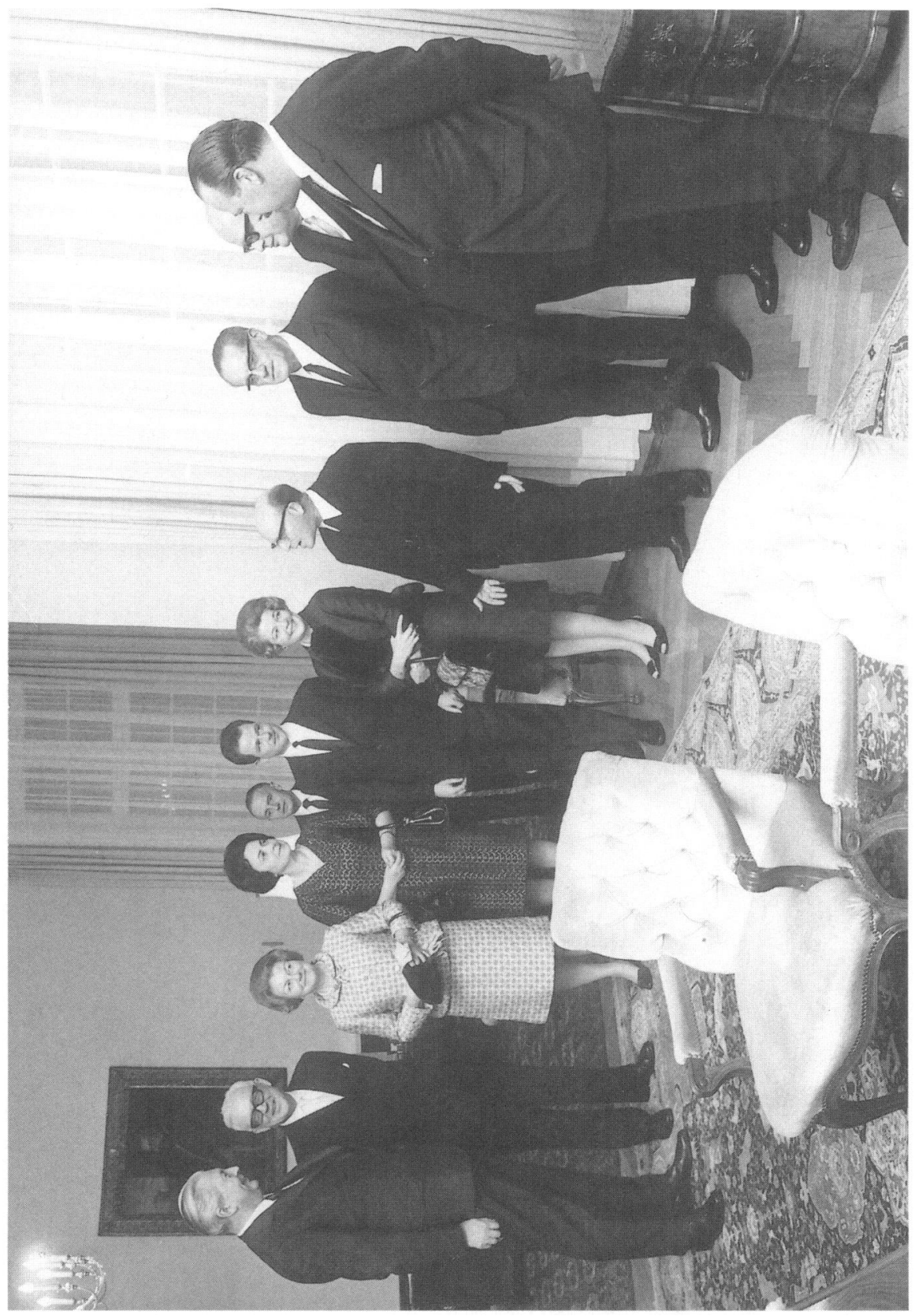

1965 Schauff im Kreis persönlicher und politischer Freunde anläßlich der Verleibung des Grofien Verdienstkreuzes mit Stern des Verdienstordens der Bundesrepublik Deutschland zu seinem 65. Geburtstag. Von links: Kurt Georg Kiesinger, Johannes und Karin Schauff, MaricLuise Kiesinger, Michael Schauff, Rosa Sophic Baronin von und zu Guttenberg, P. Paulus Gordan OSB, I lerbert Wehner, Klaus Dohrn, Karl Theodor Freiherr von und zu Guttenberg. 


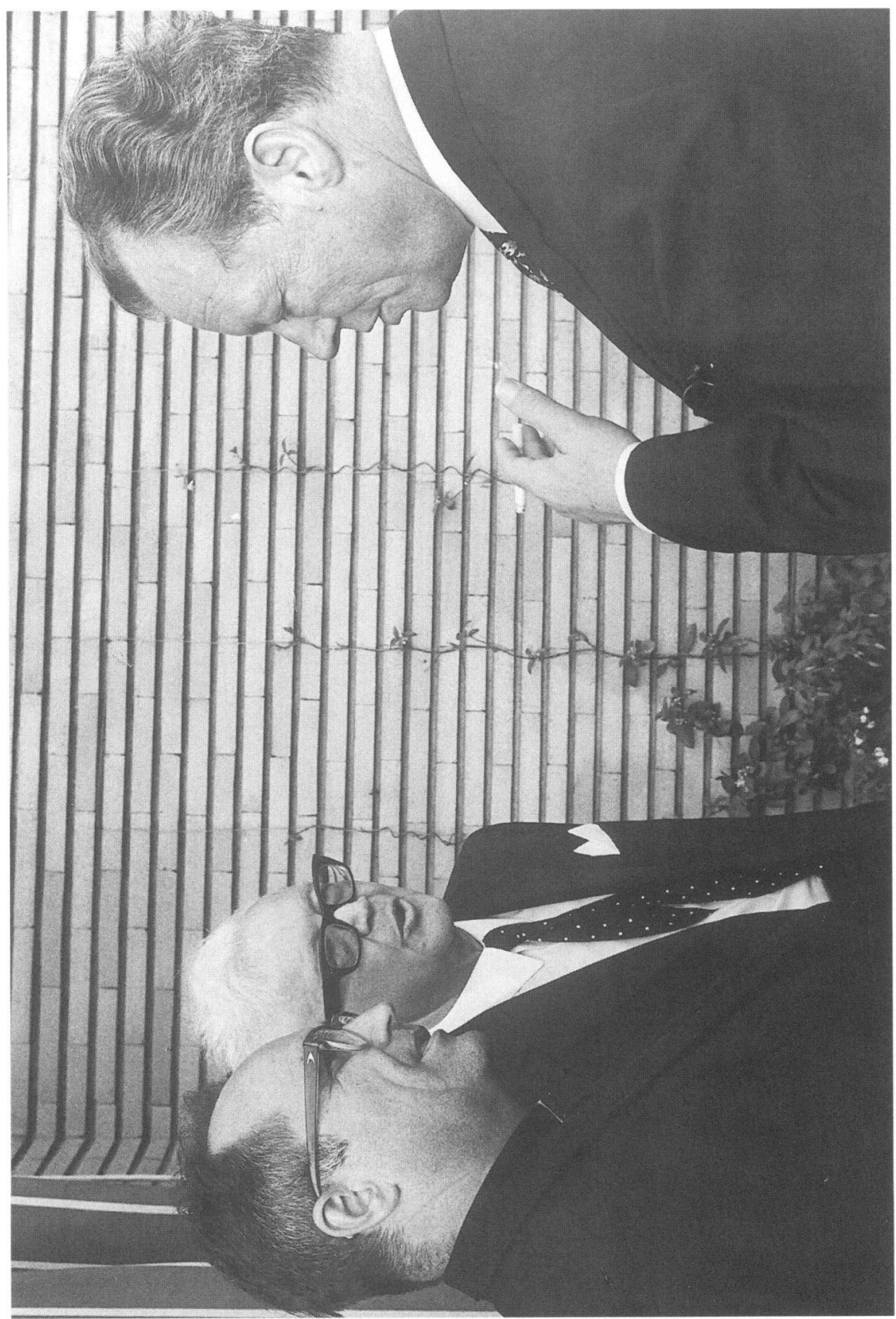

1970 Bemühungen um eine Vormalisicrung der Beziehungen der deutschen Sozialdemokratie mit der katholischen Kirche: Treften won Bundeskanzler Willy Brandt mit Johannes Schauff wahrend seines Besuchs (mit Emptang im Vatikan) in Rom. Neben Brandt und Schauff (von rechts) Mserr. Joseph Gremitlion, Generalsekretat der Kurienkommission "Justitia et Pax“. 


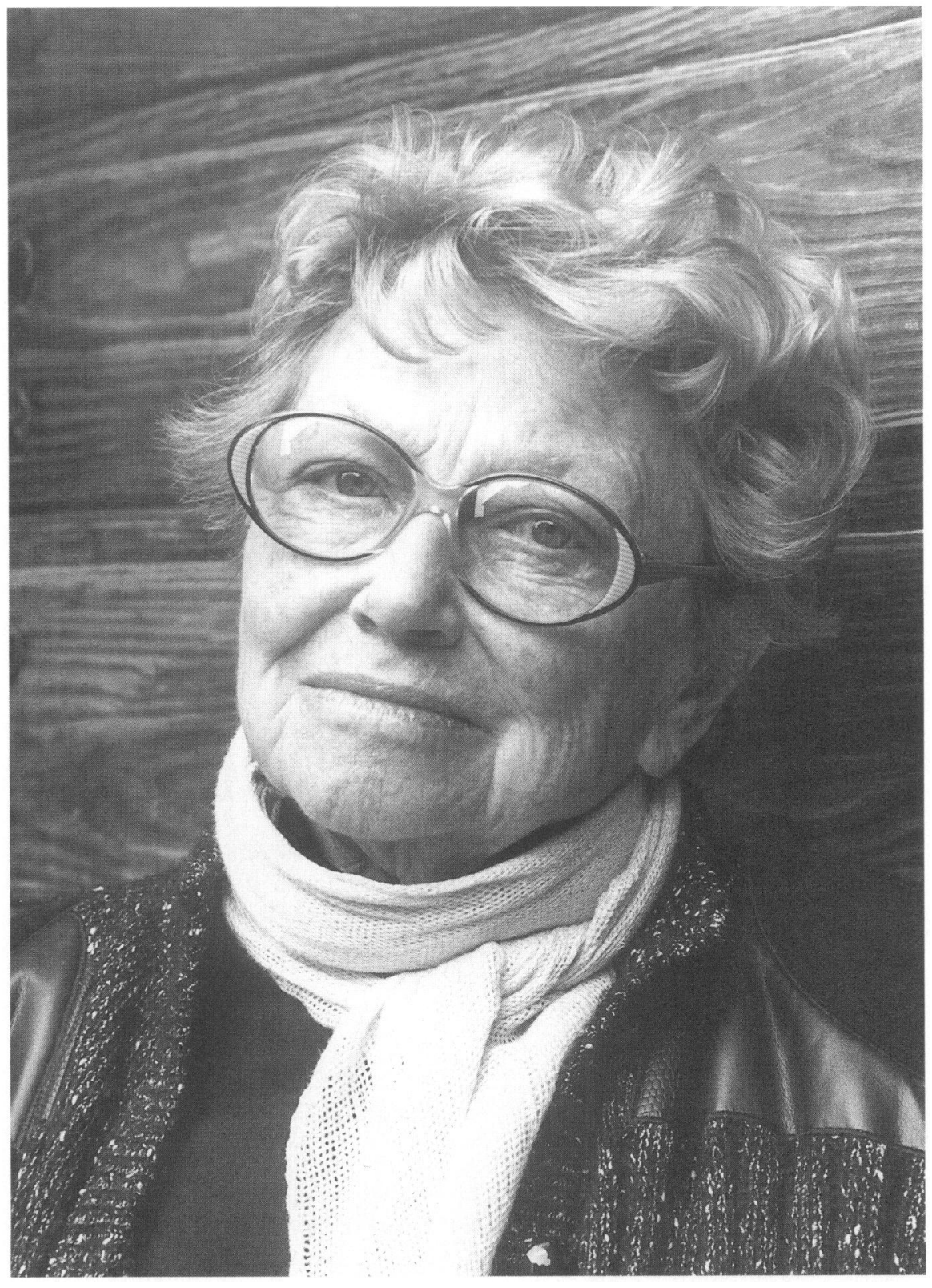




\section{Quellen und Literatur}

\section{Ungedruckte Quellen}

Institut für Zeitgeschichte, München-Berlin (IfZ)

Nachlaß Johannes Schauff

Nachlaß Dieter Sattler

Nachlaß Karl Thieme

Nachlaß Marcia Khan

Zeugenschrifttum Walther Hensel

Archiv für Christlich-Demokratische Politik der Konrad-Adenauer-Stiftung, St. Augustin (ACDP)

Nachlaß Hermann Kopf I-027

Nachlaß Heinrich Krone I-028

Nachlaß Hans Berger I-400

CDU/CSU-Bundestagsfraktion VIII-001

Archiv der sozialen Demokratie der Friedrich-Ebert-Stiftung, Bonn (AsD) Nachlaß Fritz Erler

\section{Bundesarchiv, Koblenz (BA)}

Nachlaß Karl Theodor von und zu Guttenberg

Reichstagsprotokolle (Anlagebände: Ausschuß-Protokolle), 1922-1933.

(unveröffentlichte Sammlung)

Politisches Archiv des Auswärtigen Amts, Bonn, inzwischen Berlin (PAAA)

Bestand Kulturpolitischer Beirat

Bestand Nationale Minderheiten

\section{Historisches Archiv des Erzbistums Köln}

Bestand Flüchtlings- und Vertriebenenseelsorge

\section{Katholische Universität Leuven}

Dokumente und Papiere (Nachlaß) August Vanistendael

\section{Staatsarchiv München}

Bestand Oberfinanzdirektion München (Außenhandel des Reichswirtschaftsministeriums/ Devisenbewirtschaftung) 


\section{Gedruckte Quellen}

a) Akteneditionen

Akten der Reichskanzlei. Das Kabinett von Schleicher. 3. Dezember 1932 bis 30. Januar 1933, bearb. von Anton Golecki. Boppard 1986

Akten der Reichskanzlei. Die Kabinette Brüning I und II. 30. März 1930 bis 30. Mai 1932, 3 Bde., bearb. von Tilman Koops. Bd. 1: 30. März 1930 bis 28. Februar 1931, Boppard 1982; Bd. 2: 1. März 1931 bis 10. Oktober 1931, Boppard 1982; Bd. 3: 10. Oktober 1931 bis 30. Mai 1932, Boppard 1990

Außenpolitik der Bundesrepublik Deutschland. Dokumente von 1949 bis 1994. Köln 1995

Bundes-Gesetzblatt des Norddeutschen Bundes 1867-1870, hrsg. vom Reichsministerium des Innern

Die Protokolle der Reichstagsfraktion und des Fraktionsvorstands der Deutschen Zentrumspartei 1926-1933. Bearbeitet von Rudolf Morsey. Mainz 1969

Reichsgesetzblatt 1929, Teil 1, hrsg. vom Reichsministerium des Innern. Berlin 1918, 1929, 1931. 1) Ostpreußenhilfsgesetz, Gesetz über Hilfsmaßnahmen für die notleidenden Gebiete des Ostens (Osthilfegesetz), in: RGBl. 1931, Teil 1, S. 117-122; 2) Gesetz über wirtschaftliche Hilfe für Ostpreußen, in: RGBl. 1929, Teil 1, S. 97/98

Texte zur Deutschlandpolitik. Bd. 1-2. Hrsg. Bundesministerium für gesamtdeutsche Fragen. Bonn/Berlin 1968

Verhandlungen des Deutschen Bundestags, 5. Wahlperiode, Stenographische Berichte, Bd.63, 80. Sitzung, 13. Dezember 1966, S. 3657; Bd. 70, 246. Sitzung, 2. Juli 1969, S. $13716-13719$

Verhandlungen des Reichstags, 4. Wahlperiode, Stenographische Berichte, Bd. 427, 153. Sitzung, 2. April 1930. Berlin 1930.

Widerstand als „Hochverrat“ 1933-1945. Die Verfahren gegen deutsche Reichsangehörige vor dem Reichsgericht, dem Volksgerichtshof und dem Reichskriegsgericht. MikroficheEdition und Erschließungsband, bearb. von Jürgen Zarusky und Hartmut Mehringer. München u.a. 1994-1998

Das Zweite Vatikanische Konzil. Konstitutionen, Dekrete und Erläuterungen/Kommentare, 3 Bde. (= Lexikon für Theologie und Kirche, Bde. 12-14). Freiburg-Basel-Wien 1966-1968

\section{b) Artikel und Publikationen von Johannes Schauff}

25 Jahre Rolândia. Studien zur Besiedlung des Nordens von Paraná (Hrsg.). Bonn 1957 Agrarnot und Siedlung, in: Westfälische Neueste Nachrichten (Bielefeld), 22. März 1930

Aktives und passives Wahlrecht der verschiedenen Altersgruppen, in: Der Heimatdienst, 8 (1928), Nr. 14, S. 223-225

Analyse der Konjunkturlage, in: Germania, 28. November 1926

Aus meiner beruflichen und politischen Arbeit (Dezember 1934); auszugsweise abgedruckt in: Pulheimer Beiträge zur Geschichte und Heimatkunde, Bd. 9 (1985), S. 93-94

Auswanderung nach Roland - Gründe und Hintergründe. Interview mit Johannes Schauff in der Zeitschrift „Roland“, Nr. 5, November 1957; gekürzte Fassung in: Um der Freiheit willen (Lit. über Johannes Schauff), S. 179-193

Autobiographische Notizen/Fragmente (Ms.), o. D. (1947-1980), im Besitz des Verf.

Bauerntum und Siedlung, in: Der Ost-Siedler, 3 (1932), Nr. 9, S. 1-3

Baupolitik als Konjunkturpolitik, in: Germania, 7. Oktober 1926

Bautätigkeit, in: Germania, 2. November 1926

Das Landproblem des deutschen Westens, in: Das neue Ufer, 20. März 1926

Das Wahlverhalten der deutschen Katholiken im Kaiserreich und in der Weimarer Republik.

Untersuchungen aus dem Jahre 1928 herausgegeben und eingeleitet von Rudolf Morsey. 
Mainz 1975. Erstausgabe: Die deutschen Katholiken und die Zentrumspartei. Eine politisch-statistische Untersuchung der Reichstagswahlen seit 1871. Köln 1928

Dauernde Arbeitslosigkeit, in: Germania, 12. November 1926

Der Katholizismus und das Landproblem, in: Rhein-Mainische Volkszeitung, 28. Juli 1924 und 29. Juli 1924

Der neue Großhandelsindex des Statistischen Reichsamtes, in: Handelszeitung des Berliner Tageblatts, 21. Dezember 1926

Der neueste Stand der landwirtschaftlichen Verschuldung, in: Kölnische Volkszeitung, 24. August 1926

Der Reichshaushalt 1913 und 1926, in: Das Junge Zentrum 3 (1926), H. 4, S. 78-83

Der Siedler, in: Das Junge Zentrum, 4 (1927), H. 10, S. 204-205

Deutsche Katholiken, katholische Kirche und bäuerliche Ostsiedlung. Schneidemühl o. J. (im Auftrag von Bischof Maximilian Kaller zur Vorlage auf der Fuldaer Bischofskonferenz [1930], als Manuskript gedruckt)

Deutsche und europäische Depression, in: Germania, 29. August 1926

Die Arbeitsgemeinschaft republikanischer Zentrumsstudenten, in: Republikanische Hochschulzeitung, Nr. 6, November 1925

Die Bodenreform in Chile, in: Stimmen der Zeit 88 (1962/63), Bd. 172, H. 8, S. 96-104

Die Durchführung der landwirtschaftlichen Siedlung, in: Rheinisches Land. Zeitschrift zur Pflege echter Landkultur in den Rheinlanden, H. 11/12, Februar/März 1928, S. 172-185

Die Entwicklung der Spareinlage, in: Germania, 16. Dezember 1926

Die europäische Depression, in: Dresdener Neueste Nachrichten, 25. August 1926

Die europäischen Zollmauern, in: Germania, 23. Oktober 1926

Die großdeutsche Tradition, in: Das Junge Zentrum 2 (1925), H. 1, S. 32-35 und H. 2, S. 6-10

Die innere Kolonisation in Italien (anonym), in: Der Ost-Siedler, 4 (1933), Nr. 6/7 und 10, S. 89-92 und S. 140-145

Die Jugend bei den Wahlen, in: Rhein-Mainische Volkszeitung, 20. Juli 1928 (ebenfalls erschienen in: Der Weckruf 4 [1928], Nr. 8, S. 12-13)

Die Konjukturforschung, in: Germania, 21. März 1926

Die Kreditbelastung der Landwirtschaft, in: Germania, 25. August 1926

Die Kreditlage der Landwirtschaft, in: Kölnische Volkszeitung, 20. Juni 1926 (erschienen ebenfalls in: Schlesische Volkszeitung, 31. Juli 1926; Deutsches Volksblatt, 5. August 1926; Freiburger Tagespost, 16. August 1926)

Die Kulturkrise des Landes, in: Das Junge Zentrum 4 (1927), H. 10, S. 187-190

Die Lage der Landwirtschaft seit 1924, in: Germania, 25. November 1926

Die landwirtschaftliche Erschließung Lateinamerikas und der Beitrag Europas, in: Die Neue Ordnung 15 (1961), S. 1-8

Die methodischen Grundlagen der Großhandelsindexziffernberechnung. Phil. Diss. Univ. Leipzig, 1925 (Manuskript)

Die neue Bodenreformgesetzgebung in Chile, in: Innere Kolonisation 12 (1963), H. 8, S. $170-175$

Die neue Ernte, in: Germania, 26. Oktober 1926

Die Organisation der bäuerlichen Ostsiedlung, Sonderdruck aus: Das Junge Zentrum 4 (1927), H. 10, S. 3-7

Die Ostsiedler aus dem Rheingebiet, in: Rheinischer Beobachter, Nr. 3, 1. Februarheft 1931

Die Ostsiedler aus Süddeutschland. Die Umsiedlung bis 1926, in: Der Ost-Siedler, Nr. 5/6, April/Mai 1930, S. 3-5

Die Ostsiedler aus West- und Süddeutschland, in: Archiv für innere Kolonisation XX (1928), S. 524-530

Die parteipolitische Struktur Deutschlands, in: Neues Wahlrecht, S. 139-154

Die Schicksalskurve der deutschen Zentrums-Partei, in: Main-Pfälzische Landeszeitung, 27. September 1928 
Die West-Ost-Siedlung in den Jahren 1927-1930. Veröffentlichungen der Reichsstelle für Siedlerberatung, H. 1, Berlin 1931

Einerwahlkreise?, in: Germania, 20. April 1929 (ebenfalls erschienen in Kölnische Volkszeitung, 3. Mai 1929)

Geldumlauf und bargeldloser Zahlungsverkehr, in: Germania, 22. Dezember 1926

Gesichtspunkte zur Wahlreform, in: Neues Wahlrecht, S. 200-239

Grundsätzliches zur Rücksiedlungsfrage, in: Der Ost-Siedler, 3 (1932), Nr. 5, S. 1-3

Handelsbilanzen, in: Germania, 10. August 1926

Hindernisse der West-Ostsiedlung, in: Archiv für innere Kolonisation XXII (1930), S. 237244

Interkonfessionelle Verständigung?, in: Kölnische Volkszeitung, 1. November 1932

Internationale Arbeitslosigkeit, in: Germania, 1. August 1926

Jean Mabillon. Ein Kämpfer katholischer Geschichtswissenschaft, in: Germania 28, Dezember 1922

Jungzentrum. Die Reichstagung der Windthorstbunde, in: Abendblatt der Frankfurter Zeitung, 30. August 1926

Konjunktur und Krise (unter Ps. Dr. R. Baumann), in: Der Deutsche, 23. Februar 1926 (ebenfalls erschienen in: Freiburger Tagespost, 7. Juni 1926)

Korruptionsskandale und republikanische Jugend, in: Rhein-Mainische Volkszeitung, 2. Februar 1925

Landerschließung und Kolonisation in Lateinamerika (Hrsg.). Berlin/Bonn 1959.

Landwirtschaftliche Kreditstatistik, in: Germania, 14. Juli 1926

Landwirtschaftliche Kreditstatistik, in: Germania, 20. März 1926

Motorisierung Deutschlands, in: Germania, 9. November 1926

Neue Jugend, in: Neue Jugend. Weckrufe zur Betätigung wahren Menschentums, hrsg. von G. H. Weber, Coburg. Nr. 1, April 1922

Neues Wahlrecht, in: Der Weckruf 5 (1929), Nr. 5, S. 1-2

Neues Wahlrecht. Beiträge zur Wahlreform (hrsg. von Johannes Schauff). Berlin 1929

Osthilfe und Siedlung (anonym), in: Das Zentrum, 1933, Nr. 3/4, S. 49-72

Ostsiedlung und Bauerntum, in: Der Heimatdienst, 11 (1931), Nr. 9, S. 138-140 (ebenfalls erschienen in: Die Schildgenossen, 11 [1931], Nr. 11, S. 161-168)

Parteienzersplitterung und Staatsgerichtshof, in: Germania, 4. Februar 1930

Vielleicht hätte es einige Tausend Tote gegeben. Gespräch mit Dr. Johannes Schauff, in: Guido Knopp und Bernd Wegmann, Warum habt Ihr Hitler nicht verhindert? Frankfurt a.M. 1983, S. 20-27

Volk und Volksbildung in Dänemark (zus. mit Karin Schauff). Mit einem Vorwort von Anton Heinen. Schriften des Vereins zur Förderung der Bauernbildung, H. 1. Düsseldorf o. J. (1931)

Vom jungen Zentrum. Die Reichstagung der Windthorstbunde, in: Abendblatt der Frankfurter Zeitung, 4. September 1925

Wahl als Entscheidung, in: Rhein-Mainische Volkszeitung, 27. Oktober 1924

Wahldiagnose in Köln, in: Rhein-Mainische Volkszeitung, 25. August 1929 (ebenfalls erschienen in: Der Weckruf 4 (1928), Nr. 9, S. 12)

Wahlrecht für Frauen, in: Germania, 12. November 1926

Wechsel und Konjunktur, in: Germania, 23. Dezember 1926

Wer kann siedeln? Berufskrise und Bauernsiedlung (Hrsg. ). Berlin 1932

Wie lese ich mein Konjunkturbarometer?, in: Bauwelt, H. 39, 30. September 1926

Wo steht unsere Landjugend?, in: Rheinisches Zentrum. Mitteilungen der Rheinischen Zentrumspartei, Nr. 11/12, November/Dezember 1925, Nr. 1, April 1926 sowie Nr. 2, Juli 1926 (als Manuskript gedruckt)

World Migration Movements. Book of the International Catholic Migration Congress, 12.16. Sept. 1954, Breda (Mithrsg.). The Hague 1954

Zum Problem der Massenarbeitslosigkeit, in: Germania, 5. September 1926 
Zur Konjunkturlage, in: Germania, 1. Juni 1926

Zur Soziologie der Wahlen, in: Der Heimatdienst 8 (1928), Nr. 11, S. 170 f. (ebenfalls erschienen in: Der Weckruf 4 [1928], Nr. 7, S. 1-2)

\section{c) Publikationen von Karin Schauff}

Brasilianischer Garten. Pfullingen 1970/71

Das Klingelband. Pfullingen 1979

Die Entwicklung zum Proportionalrecht in Deutschland, in: Johannes Schauff, Neues Wahlrecht, S. 126-138

Ein Sack voll Ananas. Pfullingen 1974.

Erinnerung an Dr. Carl Sonnenschein, 1876-1929, in: L'Osservatore Romano, 9. Juli 1976

Erinnerung an Ludwig Kaas. Pfullingen 1972

Heinrich von Brentano di Tremezzo. Der Mensch und Freund, in: Erbe und Auftrag, 41 (1965), hrsg. von der Erzabtei Beuron, S. 47-52

Schreib mir alles, Mutter. Pfullingen 1987

Teutones in pace. Dem Andenken an Schwester M. Magdalena Braun. Bozen 1952

Volk und Volksbildung in Dänemark (s. Schriften Johannes Schauff)

Wahlheimat Rom. Privatdruck 1968

\section{Literatur}

\section{a) Biographisches zu Johannes Schauff}

Dornseifer, Gerhard, Dr. Johannes Schauff, in Stommeln geboren - in der Welt zu Hause, in: Pulheimer Beiträge zur Geschichte und Heimatkunde, Bd. 9 (1985), hrsg. von der Stadt Pulheim. Pulheim 1985, S. 84-91

Ettighoffer, Paul C., Weiter Weg von Mecklenburg bis Brasilien! Schaffen und Schicksal eines Politikers der Weimarer Zeit, (Ms., o. J.), in: NL Schauff, Bd. 41

Exil in Brasilien. Die deutschsprachige Emigration 1933- 1945. Eine Ausstellung des Deutschen Exilarchivs 1933- 1945. Die Deutsche Bibliothek, Frankfurt a. M., hrsg. von KlausDieter Lehmann, Red. Christine Hohnschopp. Frankfurt a. M. 1994, S. 27-35

Fischer, Hans-Joachim, Ganz nach dem Herzen eines politischen Christen. Karin und Johannes Schauff - kompromißlos und menschenfreundlich, in: Frankfurter Allgemeine Zeitung, 28. Januar 1989

Fütterer, Karl, Johannes Schauff - ein Pionier der ländlichen Siedlung, Sonderheft der Schriftenreihe für ländliche Sozialfragen. Hannover 1959, S. 22-23 (auch veröffentlicht in: Innere Kolonisation 12 [1963], H. 1, S. 22-23)

Morsey, Rudolf, Ein ungewöhnliches deutsches Schicksal im 20. Jahrhundert: Johannes Schauff, in: Pulheimer Beiträge zur Geschichte und Heimatkunde, Bd. 9 (1985), S. 97-108

Ders., Johannes Schauff (1902-1990), in: Zeitgeschichte in Lebensbildern, Bd. 8: Aus dem deutschen Katholizismus des 19. und 20. Jahrhunderts, hrsg. von Jürgen Aretz, Rudolf Morsey und Anton Rauscher. Mainz 1997, S. 233-246, 322-323

Rudolph, Hermann, Ein Bauernsohn als Weltbürger, in: Die Zeit, 19. Dezember 1982

Schneider, Dieter Marc, Christliche und konservative Remigranten. Das Beispiel Johannes Schauff, in: Rückkehr und Aufbau nach 1945. Deutsche Remigranten im öffentlichen Leben Nachkriegsdeutschlands, hrsg. von Claus-Dieter Krohn und Patrik von zur Mühlen. Marburg 1997, S. 157-187

Schneider, Dieter Marc, Die Südtiroler Irredenta. Johannes Schauffs Beitrag zur Lösung eines europäischen Problems, in: „Der Fremde im Dorf“. Überlegungen zum Eigenen und 
Fremden in der Geschichte. Rex Rexheuser zum 65. Geburtstag, hrsg. von Hans-Jürgen Bömelburg und Beate Eschment. Lüneburg 1998, S. 427-444

Um der Freiheit willen. Eine Festgabe für und von Johannes und Karin Schauff zum 80 . Geburtstag, hrsg. von Paulus Gordan. Pfullingen 1983

Woller, Rudolf, Flucht in den Busch mit sieben Kindern, in: Rheinischer Merkur, 4. März 1984

\section{b) Buchpublikationen und Aufsätze}

35 Jahre Exilliteratur in der Deutschen Bibliothek Frankfurt am Main. Ein Beitrag zur Geschichte der Exilforschung in der Bundesrepublik Deutschland. Für Werner Berthold zum 31. März 1984. Frankfurt a. M. 1984

Adenauer, Konrad, Erinnerungen. Fragment. Stuttgart 1968

Alexander, Helmut/Lechner, Stefan/Leidlmair, Adolf, Heimatlos. Die Umsiedlung der Südtiroler. Wien 1993

Annuario Pontificio 1967. Città del Vaticano 1967

Anschütz, Gerhard/Richard Thoma (Hrsg.), Handbuch des deutschen Staatsrechts. Tübingen 1930

Archiv für innere Kolonisation, hrsg. von der Gesellschaft zur Förderung der inneren Kolonisation. Berlin, Jgg. 1926-1933

Bade, Klaus J., Homo migrans. Wanderungen aus und nach Deutschland. Erfahrungen und Fragen. Essen 1994

Baerwald, Friedrich, Zur politischen Tätigkeit deutscher Emigranten im Council for a Democratic Germany, in: VfZ 28 (1980), S. 372-383

Baring, Arnulf, Machtwechsel. Die Ära Brandt-Scheel. Stuttgart 1982

Barzel, Rainer, Geschichten aus der Politik. Frankfurt a. M. 1987

Bayern in der NS-Zeit. Bd. I-VI, hrsg. von Martin Broszat u.a. München 1977-1983

Becher, Herbert J., Ein früher Freund Polens, in: Um der Freiheit willen (s. unter: Biographisches zu Johannes Schauff), S. 240-246

Becker, Heinrich, Handlungsspielräume der Agrarpolitik in der Weimarer Republik zwischen 1923 und 1929. Stuttgart 1990

Becker, Josef, Zentrum und Ermächtigungsgesetz 1933 (Dokumentation), in: VfZ 9 (1961), S. $195-210$

Becker, Winfried, Politische Neuordnung aus der Erfahrung des Widerstands. Katholizismus und Union, in: Peter Steinbach (Hrsg.), Widerstand. Ein Problem aus Theorie und Geschichte, S. 261-292

Beil, Johannes, In Urwald und Großstadt Brasiliens. Ein Menschenleben im Dienst der Seelsorge und der sozialen Entwicklung. São Paulo-Aalen 1967

Bergmann, Karl Hans, Die Bewegung „Freies Deutschland in der Schweiz“ 1943-1945. München 1974

Bethell, Nicholas, Das letzte Geheimnis. Die Auslieferung russischer Flüchtlinge an die Sowjets durch die Alliierten 1944-1947. Frankfurt a. M./Berlin 1980

Bingen, Dieter, Die Polenpolitik der Bonner Republik von Adenauer bis Kohl 1949-1991. Baden-Baden 1998

Biographisches Handbuch der deutschsprachigen Emigration nach 1933/International Biographical Dictionary of Central European Emigrés, 1933-1945, hrsg. vom Institut für Zeitgeschichte, München, und der Research Foundation for Jewish Emigration, New York, unter der Ltg. von Werner Röder und Herbert A. Strauss. 3 Bde. München u.a. 1980-1983

Blumenwitz, Dieter/Klaus Gotto/Hans Maier/Konrad Repgen/Hans-Peter Schwarz (Hrsg.), Konrad Adenauer und seine Zeit. Stuttgart 1976

Boff, Leonardo, Jesus Christ Liberator. Maryknoll/NY 1978 
Boll, Bernhard/Volker Schulze/Hans Süssmuth (Hrsg. ), Zeitungsland Nordrhein-Westfalen. Geschichte - Profile - Struktur. Bonn 1993

Bonhoeffer, Dietrich, Gesammelte Schriften, hrsg. von Eberhard Bethge. Bd. VI. München 1974

Bouvier, Beatrix, Zwischen Godesberg und Großer Koalition. Der Weg der SPD in die Regierungsverantwortung. Bonn 1990

Boyens, Wilhelm Friedrich, Die Geschichte der ländlichen Siedlung. 2 Bde., hrsg. von Oswald Lehnich. Berlin-Bonn 1959-1960

Braatz, Werner E., Die agrarisch-industrielle Front in der Weimarer Republik 1930-1932, in: Schmollers Jahrbuch für Wirtschafts- und Sozialwissenschaften 91 (1971), S. 541-565

Bracher, Karl Dietrich, Die Auflösung der Weimarer Republik. Königstein/Düsseldorf 51978

Breipohl, Renate, Religiöser Sozialismus und bürgerliches Geschichtsbewußtsein zur Zeit der Weimarer Republik. Zürich 1971

Breuning, Bernd, Die deutsche Rolandwanderung (1932-1938). Soziologische Analyse in historischer, wirtschaftlicher und politischer Sicht. Mit einem Geleitwort von Johannes Schauff. München 1983

Briegel, Manfred/Wolfgang Frühwald (Hrsg.), Die Erfahrung der Fremde. Kolloquium des Schwerpunktprogramms „Exilforschung“ der Deutschen Forschungsgemeinschaft. Forschungsbericht. Weinheim 1988

Broszat, Martin, Nationalsozialistische Polenpolitik 1939-1940. Stuttgart 1961

Brüning, Heinrich, Reden und Aufsätze eines deutschen Staatsmanns, hrsg. von Wilhelm Vernekohl unter Mitwirkung von Rudolf Morsey. Münster 1968

Ders., Memoiren 1918-1934. Stuttgart 1970

Ders., Briefe und Gespräche. Bd. I: 1934-1945; Bd. II: Briefe 1946-1960, hrsg. von Claire Nix unter Mitarbeit von Reginald Phelps und George Pettee. Stuttgart 1974

Buchheim, Karl, Eine sächsische Lebensgeschichte. Erinnerungen 1889-1972. Bearbeitet von Udo Wengst u. Isabel F. Pantenburg. München 1996

Büsch, Otto/Monika Wölk/Wolfgang Wölk (Hrsg.), Wählerbewegung in der deutschen Geschichte. Analysen und Berichte zu den Reichstagswahlen 1871-1933. Berlin 1978

Camara, Helder, Revolution für den Frieden. Freiburg 1969

Corsini, Umberto/Lill, Rudolf, Südtirol 1818-1946. Bozen 1988

Courtois, Stéphane/Nicolas Werth/Jean-Louis Panné/Andrzej Paczkowski/Karel Bartosek/ Jean-Louis Margolin, Das Schwarzbuch des Kommunismus. Unterdrückung, Verbrechen und Terror. München/Zürich 1998

Czaja, Herbert, Ausgleich mit Osteuropa. Versuch einer europäischen Friedensordnung. Stuttgart 1969

Ders., Der deutsch-polnische Dialog. Ein Briefwechsel zwischen den polnischen und den deutschen Bischöfen (Vortrag vom 16. Oktober 1966). Sonderdruck der Eichendorffgilde in: H. Koschyk (Hrsg.), Herbert Czaja: Unsere sittliche Pflicht. Stuttgart 1989, S. 98-130

Ders., Unterwegs zum kleinsten Deutschland? Mangel an Solidarität mit den Vertriebenen. Marginalien zu 50 Jahren Ostpolitik. Frankfurt a.M. 1996

Der Ost-Siedler. Mitteilungsblatt der Siedlervermittlungsstelle der Gesellschaft zur Förderung der inneren Kolonisation bzw. der Reichsstelle für Siedlerberatung. Berlin 1930-1933

Deutsche Biographische Enzyklopädie, hrsg. von Walther Killy. 11 Bde. München u.a. 1995-2000

Deutsch-polnischer Dialog. Briefe der polnischen und deutschen Bischöfe und internationale Stellungnahmen. Bonn u. a. 1967

Doering-Manteuffel, Anselm, Dimensionen von Amerikanisierung in der deutschen Gesellschaft, in: Archiv für Sozialgeschichte 35 (1995), S. 1-34

Ders., Wie westlich sind die Deutschen? Amerikanisierung und Westernisierung im 20. Jahrhundert. Göttingen 1999

Dohrn, Klaus, Das Amerikabild Adenauers, in: Dieter Blumenwitz u.a. (Hrsg.), Konrad Adenauer und seine Zeit, S. 510-523 
Dörpinghaus, Bruno, Die Genfer Sitzungen führender christlich-demokratischer Politiker im Nachkriegseuropa, in: Dieter Blumenwitz u.a. (Hrsg.), Konrad Adenauer und seine Zeit, S. 538-565

Ebneth, Rudolf, Die österreichische Wochenschrift „Der christliche Ständestaat“. Deutsche Emigration in Österreich 1933-1938. Mainz 1976

Ehmke, Horst, Mittendrin. Von der Großen Koalition zur deutschen Einheit. Berlin 1994

Erdmann, Karl Dietrich, Adenauer in der Rheinlandpolitik nach dem Ersten Weltkrieg. Stuttgart 1966

Ermacora, Felix, Südtirol und das Vaterland Österreich. Wien/München 1984

Eschenburg, Theodor, Carl Sonnenschein, in: VfZ 11 (1963), S. 333-361

Falter, Jürgen W., Hitlers Wähler. München 1991

Ders./Lindenberger, Thomas/Schumann, Siegfried, Wahlen und Abstimmungen in der Weimarer Republik. Materialien zum Wahlverhalten 1919-1933. München 1986

Feilchenfeld, Werner/Dolf Michaelis/Ludwig Pinner, Haavera-Transfer nach Palästina und Einwanderung deutscher Juden 1933-1939. Tübingen 1972

Feinde werden Freunde, hrsg. von Friedbert Pflüger und Winfried Lipscher. Bonn 1993

Fiederlein, Friedrich Martin, Der deutsche Osten und die Regierungen Brüning, Papen, Schleicher. Phil. Diss. Univ. Würzburg, 1966

Forschbach, Edmund, Edgar Jung und der Widerstand gegen Hitler, in: „Civis“, 15. November 1959

Ders., Brief an Johannes Schauff, in: Um der Freiheit willen (s. unter Literatur über Johannes Schauff), S. 80-85

Ders., Edgar J. Jung. Ein konservativer Revolutionär. 30. Juni 1934. Pfullingen 1984

Framke, Gisela, Im Kampf um Südtirol. Ettore Tolomei (1865-1952) und das „Archivio per l'Alto Adige“. Tübingen 1987

Freeden, Hermann von, Meilensteine deutscher Kolonisation in Südbrasilien nach dem Ersten Weltkrieg, in: Johannes Schauff (Hrsg.), 25 Jahre Rolândia, S. 69-75

Frühwald, Wolfgang/Heinz Hürten (Hrsg.), Christliches Exil und christlicher Widerstand. Ein Symposium an der Katholischen Universität Eichstätt 1985. Regensburg 1987

Ders./Wolfgang Schieder (Hrsg.), Leben im Exil. Probleme der Integration deutscher Flüchtlinge im Ausland 1933-1945. Hamburg 1981

Fünfzehn Jahre Atlantik-Brücke (American Council on Germany). Hamburg 1967

Goergen, Hermann Mathias, Ein Leben gegen Hitler. Geschichte und Rettung der „Gruppe Goergen“. Autobiographische Skizzen. Münster u.a. 1997

Goetz, Helmut, Das Attentat in der Via Rasella (1944), in: Innsbrucker historische Studien, Bd. 6 (1983), S. 161-178

Gordan, Paulus, Freundschaft mit Bernanos. Köln/Olten 1959

Goschler, Constantin, Wiedergutmachung. Westdeutschland und die Verfolgten des Nationalsozialismus (1945-1954). München 1992

Götz von Olenhusen, Irmtraud, Jugendreich, Gottesreich, Deutsches Reich. Junge Generation, Religion und Politik 1928-1933. Köln 1987

Graß, Karl Martin, Papenkreis und Röhmkrise 1933/34. Phil. Diss. Univ. Heidelberg, 1966.

Grote, Maria, An den Ufern der Weltstadt: Ein Gedenkbuch für Dr. Carl Sonnenschein. Münster ${ }^{4} 1954$

Guttenberg, Karl Theodor Freiherr von und zu, Fußnoten. Frankfurt a. M. 1973

Hammersen, Nicolai, Politisches Denken im deutschen Widerstand. Ein Beitrag zur Wirkungsgeschichte neokonservativer Ideologien 1914-1944. Berlin 1993

Hartungen, Christoph von, u.a., Die Südtiroler Polizeiregimenter 1943-1945, in: Der Schlern. Monatszeitschrift für Südtiroler Landeskunde 55 (1981), H. 10, S. 494-516

Hehl, Ulrich von/Konrad Repgen (Hrsg.), Der deutsche Katholizismus in der zeitgeschichtlichen Forschung. Mainz 1988

Heiber, Helmut, Karl Frank und sein Reichsinstitut für Geschichte des neuen Deutschlands. Stuttgart 1966 
Heller, Edith, Macht-Kirche-Politik. Der Briefwechsel zwischen den polnischen und deutschen Bischöfen im Jahre 1965. Köln 1992

Heller, Hermann, Gesammelte Schriften, hrsg. von Martin Drath u.a. Leiden 1971

Henke, Klaus-Dietmar, Die amerikanische Besetzung Deutschlands. München 21996

Herbert, Ulrich, Fremdarbeiter. Politik und Praxis des „Ausländer-Einsatzes“ in der Kriegswirtschaft des Deutschen Reiches. Berlin/Bonn 1985

Ders., Nicht entschädigungsfähig? Die Wiedergutmachungsansprüche der Ausländer, in: Ludolf Herbst/Constantin Goschler (Hrsg.), Wiedergutmachung in der Bundesrepublik Deutschland. München 1989, S. 273-392

Herbst, Ludolf/Constantin, Goschler (Hrsg.), Wiedergutmachung in der Bundesrepublik Deutschland. München 1989

Hermens, Ferdinand A., Demokratie oder Anarchie. Untersuchungen über die Verhältniswahl. Mit einem Vorwort von Alfred Weber. Frankfurt a. M. 1951

Ders., Sicherung, Ausbau und Verankerung des parlamentarischen Systems in der Bundesrepublik, in: Verfassung und Verfassungswirklichkeit, hrsg. von Ferdinand A. Hermens, Bd. 6, Teil 1 (1972), S. 5-82

Ders., Änderung des Wahlrechts oder Ende des Parlamentarismus, in: Um der Freiheit willen (s. unter Literatur über Johannes Schauff), S. 38-60

Ders./Theodor Schieder (Hrsg.), Staat, Wirtschaft und Politik in der Weimarer Republik. Festschrift für Heinrich Brüning. Berlin 1967

Herwarth, Hans von, Von Adenauer zu Brandt. Erinnerungen. Berlin/Frankfurt a. M. 1990

Heuss, Theodor, Verhältniswahl und Parlamentarismus, in: Zeitschrift für Politik 20 (1931), S. 312-316

Ders., Friedrich Naumann. Der Mann, das Werk, die Zeit. Bd. 2. Stuttgart/Berlin 1937

Hitler, Adolf, Die Südtiroler Frage und das deutsche Bündnis-Problem. München 1926

Hitze, Guido, Die Zentrumspartei in Schlesien in der Zeit der Weimarer Republik (19191933). Magisterarbeit Gesamthochschule Wuppertal, 1992

Höfer, Josef, Rom, Römer und Romäer. Nova et vetera zum Romerlebnis im Heiligen Jahr, in: Theologie und Glaube, 1950, H. 4, S. 289-322

Hoppe, Bert, Von Schleicher zu Hitler. Dokumente zum Konflikt zwischen Reichslandbund und der Regierung Schleicher in den letzten Wochen der Weimarer Republik, in: VfZ 45 (1997), S. 629-657

Hörster-Philipps, Ulrike, Joseph Wirth (1879-1956). Eine politische Biographie. Paderborn u. a. 1998

Hürten, Heinz, Waldemar Gurian. Ein Zeuge der Krise unserer Welt in der ersten Hälfte des 20. Jahrhunderts. Mainz 1972

Ders., Zeugnis und Widerstand. Zur Interpretation des Verhaltens der katholischen Kirche im Deutschland Hitlers, in: Peter Steinbach (Hrsg.), Widerstand. Ein Problem aus Theorie und Geschichte, S. 144-159

Jacobmeyer, Wolfgang, Polnische Juden in der amerikanischen Besatzungszone (Dokumentation), in: VfZ 25 (1977), S. 120-135

Ders., Vom Zwangsarbeiter zum heimatlosen Ausländer. Die Displaced Persons in Westdeutschland 1945-1951. Göttingen 1985

Jedin, Hubert, Das Zweite Vatikanische Konzil, in: Handbuch der Kirchengeschichte, Bd. VII (Die Weltkirche im 20. Jahrhundert), hrsg. von Hubert Jedin und Konrad Repgen. Freiburg/Basel/Wien 1979, S. 97-153

Ders., Lebensbericht, hrsg. von Konrad Repgen. Mainz 1984

Katz, Robert, Mord in Rom. München 1968

Kautsky, Karl, Die Agrarfrage. Stuttgart 1904

Kiesinger, Kurt Georg, Dunkle und helle Jahre. Erinnerungen 1904-1958, hrsg. von Reinhard Schmoeckel. Stuttgart 1989

Klemperer, Klemens von, Glaube, Religion, Kirche und der deutsche Widerstand gegen den Nationalsozialismus, in: VfZ 28 (1980), S. 239-309 
Koch-Weser, Erich, Hitler and Beyond. A German Testament. A. Knopf (Ed.). New York 1945

Koenen, Andreas, Der Fall Carl Schmitt. Sein Aufstieg zum „Kronjuristen des Dritten Reiches". Darmstadt 1995

Koestler, Arthur, Darkness at Noon. London 1940 (deutsche Ausgabe: Sonnenfinsternis. Stuttgart 1948)

Köhler, Henning, Adenauer und die rheinische Republik. Der erste Anlauf 1918-1924. Opladen 1986

Kohlhepp, Gerd, Agrarkolonisation in Nord-Paraná. Wirtschafts- und sozialgeographische Entwicklungsprozesse einer randtropischen Pionierzone Brasiliens unter dem Einfluß des Kaffeeanbaus. Wiesbaden 1975

Kosminsky, Ethel V., Rolândia, a terra prometida. São Paulo 1985

Kosthorst, Daniel, Brentano und die deutsche Einheit. Die Deutschland- und Ostpolitik des Außenministers im Kabinett Adenauer 1955-1961. Düsseldorf 1993

Krieger, Silke (Hrsg.), Partner für den demokratischen Weg. St. Augustin 1983

Kroegel, Dirk, Einen Anfang finden! Kurt Georg Kiesinger in der Außen- und Deutschlandpolitik der Großen Koalition. München 1997

Krohn, Claus-Dieter/Patrik von zur Mühlen (Hrsg.), Rückkehr und Aufbau nach 1945. Deutsche Remigranten im öffentlichen Leben Nachkriegsdeutschlands. Marburg 1997

Krone, Heinrich, Tagebücher. Erster Band: 1945-1961, bearb. von Hans-Otto Kleinmann. Düsseldorf 1995

Kronstein, Heinrich, Briefe an einen jungen Deutschen. München 1967

Kühnhardt, Ludger, Die Flüchtlingsfrage als Weltordnungsproblem. Wien 1984

Küppers, Heinrich, Joseph Wirth. Parlamentarier, Minister und Kanzler der Weimarer Republik. Stuttgart 1997

Lamesfeld, Jean, La Roque sur Pernes - eine siedlerische Glanzleistung von Banater Bauern in Frankreich, in: Jahrbuch der Donauschwaben aus Jugoslawien: Patenschaftskalender 1968. Sindelfingen 1968, S. 57-62

Ders., Von Österreich nach Frankreich: Die Banater Aktion und Robert Schuman. Salzburg 1973

Latour, Conrad F., Südtirol und die Achse Berlin-Rom 1938-1945. Stuttgart 1962

Lehmann, Klaus-Dieter (Hrsg.) und Christine Hohnschopp (Red.), Exil in Brasilien. Die deutschsprachige Emigration 1933-1945. Eine Ausstellung des Deutschen Exilarchivs 1933-1945. Deutsche Bibliothek Frankfurt a. M./Leipzig 1994

Lücke, Paul, Ist Bonn doch Weimar? Der Kampf um das Mehrheitswahlrecht. Frankfurt a. M./Berlin 1968

M. d. R. Die Reichstagsabgeordneten der Weimarer Republik in der Zeit des Nationalsozialismus. Politische Verfolgung, Emigration und Ausbürgerung 1933-1945, hrsg. von Martin Schumacher. Düsseldorf ${ }^{3} 1994$

Maier, Max Hermann, Den Baumeistern Rolândias zum Gedächtnis, in: Roland. Das monatliche Mitteilungsblatt von Pro-Arte-Rolândia, Nr. 5, November 1957, S. 1-2

Ders., Ein Frankfurter Rechtsanwalt wird Kaffeepflanzer im Urwald Brasiliens. Bericht eines Emigranten 1938-1975. Frankfurt a. M. 1975

Marquardt-Bigman, Petra, Amerikanische Geheimdienstanalysen über Deutschland 19421949. München 1995

Martiny, Martin, Die Entstehung und politische Bedeutung der „Neuen Blätter für den Sozialismus " und ihres Freundeskreises, in: VfZ 25 (1977), S. 373-419

Matthias, Erich/Rudolf Morsey (Hrsg.), Das Ende der Parteien 1933. Düsseldorf 1960

May, Georg, Ludwig Kaas. Der Priester, der Politiker und der Gelehrte aus der Schule von Ulrich Stutz. 3 Bde. Amsterdam 1981-1982

Mehringer, Hartmut, Waldemar von Knoeringen. Der Weg vom revolutionären Sozialismus zur sozialen Demokratie. München 1989 
Ders., Impulse sozialdemokratischer Remigranten auf die Modernisierung der SPD, in: Krohn/zur Mühlen (Hrsg.), Rückkehr und Aufbau nach 1945, S. 91-110

Ders., Widerstand und Emigration. Das NS-Regime und seine Gegner. München 1997

Ders./Röder, Werner/Schneider, Dieter Marc, Zum Anteil ehemaliger Emigranten am politischen Leben der Bundesrepublik Deutschland, der Deutschen Demokratischen Republik und der Republik Österreich, in: Frühwald/Schieder (Hrsg.), Leben im Exil, S. 207-223

Merkenich, Stephanie, Grüne Front gegen Weimar. Reichs-Landbund und agrarischer Lobbyismus 1918-1933. Düsseldorf 1998

Mierendorff, Carl(o), Lebendige Demokratie, in: Deutsche Republik 3 (1929), H. 45, S. 1401-1404

Möller, Horst, Gottfried Reinhold Treviranus. Ein Konservativer zwischen den Zeiten, in: Um der Freiheit willen (s. unter: Biographisches zu Johannes Schauff), S. 118-146

Ders., Parlamentarismus in Preußen 1919-1932. Düsseldorf 1985

Ders., Der Begriff der Nation und die ethnische Mobilität in historischer Perspektive, in: Das Individuum und die gemeinschaftlichen Beziehungen in Europa an der Schwelle des dritten Jahrtausends. Akten der XXIII. internationalen Tagung deutsch-italienischer Studien. Meran 1996, S. 332-353

Ders., Weimar. Die unvollendete Demokratie. München 61997

Mommsen, Hans, Gesellschaftsbild und Verfassungspläne des deutschen Widerstandes, in: Der deutsche Widerstand gegen Hitler. Vier historisch-kritische Studien von Hermann Graml, Hans Mommsen, Hans-Joachim Reichhardt und Ernst Wolf, hrsg. Walter Schmitthenner und Hans Buchheim. Köln/Berlin 1966, S. 73-167

Morsey, Rudolf, Die Deutsche Zentrumspartei, in: Matthias/Morsey (Hrsg.), Das Ende der Parteien, S. 282-453

Ders., Hitlers Verhandlungen mit der Zentrumsführung am 31. Januar 1933 (Dokumentation), in: VfZ 9 (1961), S. 182-194

Ders., Der Untergang des politischen Katholizismus. Die Zentrumspartei zwischen christlichem Selbstverständnis und „Nationaler Erhebung“ 1932/33. Stuttgart-Zürich 1977

Ders., Leben und Überleben im Exil. Am Beispiel von Joseph Wirth, Ludwig Kaas und Heinrich Brüning, in: Um der Freiheit willen (s. unter: Biographisches zu Johannes Schauff), S. 86-117

Ders., Brünings Einschätzung der politischen Entwicklung in Deutschland 1934-1948, in: Frühwald/Hürten (Hrsg.), Christliches Exil und christlicher Widerstand, S. 371-393.

Ders., Georg Schreiber, in: Wolfgang Treue/Karlfried Gründer (Hrsg.), Berlinische Lebensbilder: Wissenschaft in Berlin. Berlin 1987, S. 269-284

Ders., Die Vorbereitung der Großen Koalition von 1966. Unionspolitiker im Zusammenspiel mit Herbert Wehner, in: Von der Arbeiterbewegung zum modernen Sozialstaat. Festschrift für Gerhard A. Ritter zum 65. Geburtstag, hrsg. Jürgen Kocka, Hans-Jürgen Puhle und Klaus Tenfelde. München u. a. 1994, S. 462-478

Ders., Gründung und Gründer der Kommission für Zeitgeschichte 1960-1962, in: Historisches Jahrbuch 115 (1995), S. 453-485

Ders., Heinrich Lübke. Eine politische Biographie. Paderborn u. a. 1996

Ders., Brüning und Adenauer. Zwei Wege deutscher Politik im 20. Jahrhundert. Eine Forschungsbilanz nach 25 Jahren. Speyrer Vorträge/Hochschule für Verwaltungswissenschaften Speyer, 1996

Ders./Konrad Repgen (Hrsg.), Adenauer-Studien, Bd. I. Mainz 1971

Müller, Frank, Die „Brüning Papers“: Der Nachlaß des letzten Zentrumskanzlers in Harvard, in: Historisches Jahrbuch 1993

Müller, Hermann, Die Novemberrevolution. Berlin 1928

Muth, Heinrich, Agrarpolitik und Parteipolitik im Frühjahr 1932, in: Hermens/Schieder (Hrsg.), Staat, Wirtschaft und Politik in der Weimarer Republik, S. 317-360

Neue Deutsche Biographie, hrsg. von der Historischen Kommission bei der Bayerischen Akademie der Wissenschaften (bisher Bd. 1-19). Berlin $1953 \mathrm{ff}$. 
Neufeldt, Hans-Joachim/Huck, Jürgen/Tessin, Georg, Zur Geschichte der Ordnungspolizei 1936-1945. Koblenz 1957

Nixdorf, Oswald, Pionier im brasilianischen Urwald. Tübingen 1979

Opitz, Peter J., Das Weltflüchlingsproblem. Ursachen und Folgen. München 1988

Pailer, Wolfgang, Stanislaw Stomma. Nestor der deutsch-polnischen Verständigung. Bonn 1995

Petersen, Jens, Vorspiel zu "Stahlpakt“ und Kriegsallianz. Das deutsch-italienische Kulturabkommen vom 23. November 1938, in: VfZ 36 (1988), S. 41-77

Pflüger, Friedbert/Winfried Lipscher (Hrsg.), Feinde werden Freunde. Von den Schwierigkeiten der deutsch-polnischen Nachbarschaft. Bonn 1993

Plum, Günter, Gesellschaftsstruktur und politisches Bewußtsein in einer katholischen Region 1928-i933. Untersuchungen am Beispiel des Regierungsbezirks Aachen. Stuttgart 1972

Pohl, Heinrich, Das Reichstagswahlrecht, in: Gerhard Anschütz/Richard Thoma (Hrsg.), Handbuch des deutschen Staatsrechts, Bd. 1. Tübingen 1930, S. 386-400

Portelli, Hugues/Jansen, Thomas, La Démocatie chrétienne, force internationale. Nanterre 1986

Portner, Ernst, Koch-Wesers Verfassungsentwurf. Ein Beitrag zur Ideengeschichte der deutschen Emigration, in: VfZ 14 (1966), S. 280-298

Prauser, Steffen, Die sogenannten Geiselerschießungen in den Fosse Ardeantine im Rahmen der deutschen Besatzungspolitik in Rom. Magisterarbeit (Ms.) Univ. Frankfurt a. M. 1998

Prehn, Helmut, Das Wesen der auf der Grundlage des Reichssiedlungsgesetzes vom 11. August 1919 betriebenen Siedlungspolitik der herrschenden Klasse in der Zeit der Weimarer Republik. Diss. Universität Rostock 1969

Prüser, Friedrich, Roland und Rolândia. Bremen 1957

Pufendorf, Astrid von, Otto Klepper (1888-1957). Deutscher Patriot und Weltbürger. München 1997

Radkau, Joachim, Die deutsche Emigration in den USA. Ihr Einfluß auf die amerikanische Europapolitik 1933-1945. Düsseldorf 1971

Rahner, Hugo (Hrsg.), Befreiende Theologie der Gegenwart. Stuttgart 1977

Renger, Annemarie, Ein politisches Leben. Erinnerungen. Stuttgart 1993

Repgen, Konrad, 25 Jahre Kommission für Zeitgeschichte - ein Rückblick, in: Hehl/Repgen (Hrsg.), Der deutsche Katholizismus in der zeitgeschichtlichen Forschung, S. 9-17

Reutter, Lutz-Egon, Katholische Kirche als Fluchthelfer im Dritten Reich. Die Betreuung von Auswanderern durch den St. Raphaels-Verein. Recklinghausen-Hamburg 1971

Riesenberger, Dieter, Die katholische Friedensbewegung in der Weimarer Republik. Düsseldorf 1976

Röder, Werner, Die Dokumentation zur Emigration 1933-1945, in: Internationale Wissenschaftliche Korrespondenz zur Geschichte der Arbeiterbewegung, H. 11/12 (April 1971), S. 54-57

Ders./Hermann Weiß/Klaus A. Lankheit, Das Archiv des Instituts für Zeitgeschichte, in: 50 Jahre Institut für Zeitgeschichte. Eine Bilanz. Hrsg. von Horst Möller und Udo Wengst. München 1999, S. 105-125

Roland. Mitteilungsblatt Pro-Arte-Rolândia (1957)

Rupieper, Hermann-Josef, Die Wurzeln der westdeutschen Nachkriegsdemokratie. Der amerikanische Beitrag 1945-1952. Opladen 1993

Sachwörterbuch der Geschichte Deutschlands und der deutschen Arbeiterbewegung. 2 Bde. Berlin (-Ost) 1970

Saint-Sauveur-Henn, Anne (Hrsg.), Zweimal verjagt. Die deutschsprachige Emigration und der Fluchtweg Frankreich - Lateinamerika 1933-1945. Berlin 1998

Santel, Bernhard, Migration in und nach Europa. Erfahrungen, Struktur, Politik. Opladen 1995

Scarano, Federico, Mussolini e la Repubblica di Weimar. Le relazioni diplomatiche fra Italia e Germania. Neapel 1996 
Schaefer, Karl Heinz, Der Beratungsausschuß für Umsiedlungsgeschädigte 1964 bis 1999, Redems. (im Besitz des Verf.)

Schanbacher, Eberhard, Parlamentarische Wahlen und Wahlsystem der Weimarer Republik. Düsseldorf 1992

Schechtman, Joseph B., European Population Transfers. New York 1946

Schellenberger, Barbara, Katholische Jugend und Drittes Reich. Mainz 1975

Schirilla, Laszlo, Wiedergutmachung für Nationalgeschädigte. Ein Bericht über die Benachteiligung von Opfern der nationalsozialistischen Gewaltherrschaft. München 1982

Schmid, Carlo, Erinnerungen. München 1981

Schmidlin, Joseph, Geschichte der deutschen Nationalkirche in Rom. Freiburg/Br. 1906

Schneider, Dieter Marc, Saarpolitik und Exil, in: VfZ 25 (1977), S. 467-545

Ders., „.. . Ein Land der Zukunft“. Deutschsprachige Emigranten in Brasilien nach 1933, in:

Um der Freiheit willen (s. unter: Biographisches zu Johannes Schauff), S. 147-178

Scholder, Klaus, Die Kirchen und das Dritte Reich. 2 Bde. Frankfurt a. M./Berlin 1977-1985

Scholz, Rudolf, Deutsche Entwicklungspolitik. Eine Bilanz nach 25 Jahren. München 1979

Schröder, Wilhelm, Handbuch der sozialdemokratischen Parteitage. Bd. 1: 1863 bis 1909.

Tübingen 1930

Schulthess' Europäischer Geschichtskalender (Neue Folge), Bd. 73 (1932), München 1933

Schulz, Gerhard, Zwischen Demokratie und Diktatur. Verfassungspolitik und Reichsreform in der Weimarer Republik, Bd. 3: Von Brüning zu Hitler. Der Wandel des politischen Systems in Deutschland. Berlin/New York 1992.

Schwarz, Hans-Peter, Adenauer. Der Aufstieg 1876-1952. Stuttgart 1986

Ders., Adenauer. Der Staatsmann 1952-1967. Stuttgart 1991

Ders., Vom Reich zur Bundesrepublik. Deutschland im Widerstreit der außenpolitischen Konzeptionen in den Jahren der Besatzungsherrschaft 1945-1949. Neuwied/Berlin 1966 Schwarz, Jürgen, Studenten in der Weimarer Republik. Die deutsche Studentenschaft in der Zeit von 1918 bis 1923 und ihre Stellung zur Politik. Berlin 1971

Schwerin, Friedrich von, Die Bedeutung der Grundbesitzverteilung vom nationalen Standpunkt aus. Lissa 1913

Senz, Ingomar, Die Donauschwaben. München 1994

Seraphim, Hans-Jürgen (Hrsg.), Deutsche Siedlungsbank 1930-1960. Münster 1960

Shuster, George N., In Amerika und Deutschland. Erinnerungen eines amerikanischen College-Präsidenten. Frankfurt a. M. 1965

Smolka, Georg, Die Auswanderung als politisches Problem in der Ära des Deutschen Bundes (1815-1866), hrsg. vom Forschungsinstitut für öffentliche Verwaltung bei der Hochschule für Verwaltungswissenschaften Speyer. Speyer 1993

Soell, Hartmut, Fritz Erler - Eine politische Biographie. 2 Bde. Berlin/Bonn/Bad Godesberg 1976

Ders., Der junge Wehner. Zwischen revolutionärem Mythos und politischer Vernunft. Stuttgart 1991

Solchany, Jean, Vom Antimodernismus zum Antitotalitarismus. Konservative Interpretationen des Nationalsozialismus in Deutschland 1945-1949, in: VfZ 44 (1996), S. 373-394

Stehle, Hansjakob, Der Vatikan und die Oder-Neiße-Grenze, in: Europa Archiv, 27 (1972), S. 559-566

Ders., Der Briefwechsel der Kardinäle Wyszynski und Döpfner im deutsch-polnischen Dialog von 1970-1971, in: VfZ 31 (1983), S. 536-553

Ders., Johannes Schauff - ein Vermittler deutsch-polnischer Verständigung, in: Um der Freiheit willen (s. unter: Biographisches zu Johannes Schauff), S. 247-253

Ders., Seit 1960: Der mühsame katholische Dialog über die Grenze, in: Ungewöhnliche Normalisierung, hrsg. von Werner Plum. Bonn 1984, S. 155-178

Steinbach, Peter (Hrsg.), Widerstand. Ein Problem zwischen Theorie und Geschichte. Köln 1987 
Steininger, Rolf, Los von Rom? Die Südtirolfrage 1945/46 und das Gruber-De GasperiAbkommen. Innsbruck 1987

Ders., Südtirol zwischen Diplomatie und Terror 1947-1969. 3 Bde. Bozen 1999

Strobel, Georg W., Die Wirtschaftsbeziehungen Deutschlands und Polens 1919-1932, in: Die deutsch-polnischen Beziehungen 1919-1932. XVII. deutsch-polnische Schulbuchkonferenz der Historiker vom 11. bis 17. Juni 1984 in Augsburg. Braunschweig 1985

Ders., Nationalitätenprobleme in Ostmitteleuropa. Räte-Großdeutschland als Mittel kommunistischer Revolutionsstrategie, in: Hans Hecker/Silke Spieler (Hrsg.), Nationales Selbstverständnis und politische Ordnung. Abgrenzungen und Zusammenleben in Ostmitteleuropa bis zum Zweiten Weltkrieg. Bonn 1991

Ders., Die polnische "Preußenkrankheit" und ihre politische Instrumentalisierung, in: Aus Politik und Zeitgeschichte, B 53/1997, S. 21-33

Topf, Erwin, Die grüne Front. Der Kampf um den deutschen Acker. Berlin 1933

Toscano, Mario, Storia diplomatica della questione dell'Alto Adige. Bari 1968

Tucci Carneiro, Maria Luiza, O Anti-Semitismo na era Vargas. Fantasmas de uma geração (1930-1945). São Paulo 1988

Unterrichter, Michael von, Lo status dei residenti Alto Altesini alla luce degli accordi internazionali tra Italia, Germania e Austria. Tesi di Laurea Univ. Urbino, 1982

Unterrichter, Rudolf von, Südtirol im Blick auf Europa. Die deutsch-italienischen Umsiedlungsverträge des Jahres 1933, Auswirkungen und Lösungen, in: Um der Freiheit willen (s. unter Literatur über Johannes Schauff), S. 225-239

Verfassung und Verfassungswirklichkeit. Hrsg. Ferdinand A. Hermens. Köln/Opladen $1966 \mathrm{ff}$.

Versöhnung aus der Kraft des Glaubens. Analysen, Dokumente, Perspektiven. 1965-1985: 20 Jahre seit dem Briefwechsel zwischen den polnischen und deutschen Bischöfen, hrsg. von der Pressestelle der Deutschen Bischofskonferenz, Red. Gerhard Albert, Rudolf Hammerschmidt und Reinhold Lehmann. Bonn 1985

Vockel, Heinrich, Wahlrechtsreform, in: „Das Junge Zentrum“ ${ }^{\circ} 6$ (1929), S. 73-78

Voigt, Klaus, Zuflucht auf Widerruf. Exil in Italien 1933-1945. 2 Bde. Stuttgart 1989-1993

Ders., Der Vatikan und die Brasilien-Visa 1940-1941, in: Saint-Sauveur-Henn (Hrsg.), Zweimal verjagt, S. 66-75

Volk, Ludwig, Das Reichskonkordat vom 20. Juli 1933. Mainz 1972

Waal, Anton de, Der Campo Santo der Deutschen zu Rom. Freiburg/Br. 1896

Wehler, Hans-Ulrich, Zum Verhältnis von Geschichtswissenschaft und Psychoanalyse, in: HZ 208 (1969), S. 529-554

Wengst, Udo, Schlange-Schöningen, Ostsiedlung und die Demission der Regierung Brüning, in: GWU 30 (1979), Nr. 9, S. 538-551

Widerstand, Verfolgung und Emigration 1933-1945 (Ms., hrsg. vom Forschungsinstitut der Friedrich-Ebert-Stiftung). Bad Godesberg 1967

Widmann, Horst, Exil und Bildungshilfe. Die deutschsprachige akademische Emigration in die Türkei nach 1933. Bern/Frankfurt a. M. 1973

Wirth, Joseph, Eine offene Antwort, in: Deutsche Republik, 3 (1928), S. 706-710

Wirz, Ulrich, Karl Theodor von und zu Guttenberg und das Zustandekommen der Großen Koalition. Grub am Forst 1997

Witt, Peter-Christian, Friedrich Ebert. Parteiführer, Reichskanzler, Volksbeauftragter, Reichspräsident. Bonn 1987

Wittfogel, Karl A. , Die orientalische Despotie. Eine vergleichende Untersuchung totaler Macht. Köln/Berlin 1962

Yzermans, Vincent A., The people I love. A Biography of Luigi G. Ligutti. Collegeville/ Minn. 1976

Zur Mühlen, Patrik von, Fluchtziel Lateinamerika. Die deutsche Emigration 1933-1945: Politische Aktivitäten und soziokulturelle Integration. Bonn 1988 


\section{Abkürzungsverzeichnis}

ACDP

AFL/CIO

ASA

AsD

BA

BEG

BGBl

BHB

BMF

BVP

BWZ

CDU

CIDSE

CSU

CTNP

$\mathrm{CV}$

DC

DDP

DDR

DJK

DNVP

DP

FAO

FAZ

FDP

FIS

FUI

GCIE

GFK

GHS

GWU

$\mathrm{HZ}$

IBFG

ICEM

ICMC

ICMLF

IFCTU

IfZ

IIS

ILO
Archiv für Christlich-Demokratische Politik der Konrad-AdenauerStiftung

American Federation of Labor/Congreß of Industrial Organisations Accion Social Cattolica

Archiv für soziale Demokratie der Friedrich-Ebert-Stiftung

Bundesarchiv

Bundesentschädigungsgesetz

Bundesgesetzblatt

Biographisches Handbuch der deutschsprachigen Emigration

Bundesministerium der Finanzen

Bayerische Volkspartei

Bundesministerium für wirtschaftliche Zusammenarbeit

Christlich-Demokratische Union Deutschlands

Coopération internationale pour le développement socio-économique Christlich-Soziale Union

Companhia de Terras Norte do Paraná

Kartellverband der Katholischen Deutschen Studenten-Verbindungen

Democrazia Cristiana

Deutsche Demokratische Partei

Deutsche Demokratische Republik

Deutsche Jugendkraft

Deutschnationale Volkspartei

Displaced Person

Food and Agriculture Organisation

Frankfurter Allgemeine Zeitung

Freie Demokratische Partei

Fondation Internationale de Solidarité

Fédération Universitaire Internationale pour la Société des Nations

Giunta Cattolica Italiana per l'Emigrazione

Gesellschaft zur Förderung der inneren Kolonisation

Gesamthochschule

Geschichte in Wissenschaft und Unterricht

Historische Zeitschrift

Internationaler Bund Freier Gewerkschaften

Intergovernmental Committee for European Migration

International Catholic Migration Commission

International Catholic Migrant Loan Fund

International Federation of Christian Trade Unions

Institut für Zeitgeschichte

Institut für Internationale Solidarität

International Labour Organisation/Office 
INES

IRO

ISK

KPD

KPDO

\section{LAG}

LH

$\mathrm{MdB}$

$\mathrm{MdR}$

Msgr.

NCWC

NDB

NEI

NSDAP

OFM

OMGUS

OSB

OSS

PAAA

PDC

RGBl.

RM

RT

SA

SAPD

SJ

SPD

SS

SSS

SVP

UMDC UNESCO

UNO

UNRRA

USPD

VfZ

WK

WRS
Instituto Nacional de Estudios Sindicales (Venezuela)

International Refugee Organisation

Internationaler Sozialistischer Kampfbund

Kommunistische Partei Deutschlands

Kommunistische Partei Deutschlands/Opposition

Lastenausgleichsgesetz

Lufthansa

Mitglied des Bundestags

Mitglied des Reichstags

Monsignore

National Catholic Welfare Conference

Neue Deutsche Biographie

Nouvelles Equipes Internationales

Nationalsozialistische Deutsche Arbeiterpartei

Ordo Fratrum Minorum (Franziskaner)

Office of Military Government (for Germany), USA

Ordo Sancti Benedicti

Office of Strategic Services, USA

Politisches Archiv de Auswärtigen Amtes

Partie Démocrate Chrétienne

Reichsgesetzblatt

Reichsmark

Reichstag

Sturmabteilung der NSDAP

Sozialistische Arbeiterpartei Deutschlands

Societas Jesu (Jesuitenorden)

Sozialdemokratische Partei Deutschlands

Schutzstaffel der NSDAP

Sekretariat Sozialer Studentenarbeit

Südtiroler Volkspartei

Union Mondiale Démocrate Chrétienne

United Nations Educational, Scientific and Cultural Organisation

United Nations Organisation

United Nations Relief and Rehabilitation Administration

Unabhängige Sozialdemokratische Partei Deutschlands

Vierteljahrshefte für Zeitgeschichte

Wahlkreis

War Relief Services 


\section{Personenregister}

Auf die Aufname der Namen Karin und Johannes Schauff sowie ihrer Kinder wurde verzichtet.

Acheson, Dean 134

Adam, Wilhelm 66

Adenauer, Konrad $97 \mathrm{f}$., 139, 141, $145 \mathrm{f}$., $152,175,191,197,211 \mathrm{f} ., 214$

Aereboe, Friedrich 82

Albertz, Heinrich 176

Albrecht, Dieter $196 \mathrm{ff}$.

Althammer, Walter 140

Altmann, Oskar 82

Andreotti, Giulio 119

Arndt, Adolf 159, 192

Arnold 75

Arnold, Hans 179

Arnold, Karl 68, 97, 99

Bachmann 69

Baerwald, Friedrich 209

Baldwin, Roger 210

Ball, George W. 134

Baring, Arnulf 150, 154

Barzel, Rainer 149, 157, 162

Battaglia, Edoardo 118

Bauer, Clemens 189, 192, 198

Bea, Augustin 160, $194 \mathrm{f}$.

Becher, Herbert 187

Becher, Ulrich 85

Beck, Ludwig 68

Beil, Johannes 71,73

Bekessy, Hans $133 \mathrm{f}$.

Bell, Hans 54

Benelli, Giovanni $161,171,184$

Bergengruen, Werner 16

Berger, Hans 14, 153f., 158, 161 f., 197

Bergstraesser, Arnold 134 f., 138, 205

Berloffa, Alcide 119

Bernanos, Georges 88

Bernardini, Felippe 84

Bernassola, Angelo $141 \mathrm{f}$.

Berning, August H. 83, 193 f., 196

Berning, Wilhelm 28, 83

Bertram, Adolf 62

Beyerle, Josef 196

Bidault, Georges 131

Birrenbach, Kurt 134, 157

Blankenhorn, Herbert 133

Blomberg, Werner von 66

Blumenfeld, Erik 134
Bock, Fedor von 66

Bockius, Fritz 60

Böhler, Wilhelm $190 \mathrm{ff}$.

Böker, Alexander 133, 210

Bolz, Eugen $60 \mathrm{f}$.

Bonhoeffer, Dietrich 204

Borkenau, Franz 208

Bormann, Martin 113

Bose, Herbert von 65,67

Bosl, Karl 198

Boyens, Wilhelm 30, 99, 136

Bracher, Karl Dietrich 192

Brandt, Karl 132

Brandt, Willy 156ff., 161, 181 f., 201

Braubach, Max 194, 197

Brauer, Max 134, 208

Braun, Otto 132, 155, 211

Brauns, Heinrich 51

Bredow, Ferdinand von 67

Bredow, Kurt von 66

Brentano, Heinrich von $14,97,118,129$, $134 \mathrm{f} ., 145 \mathrm{f}$., 175 f., $179,182 \mathrm{f} ., 187$

Bresslau, Hermann Miguel 82

Briefs, Goetz $206 \mathrm{f}$.

Briemle, Theodosius 84

Broederich, Silvio 27-30, 32

Broszat, Martin 212

Brüning, Heinrich 11, 14, 20, 23 f., 31, $33 \mathrm{ff}$., 48, 50-61, 66, 69, 71, 87, $97 \mathrm{f}$., 133 , 145, 190 f., 193, 197, 200, 205f., 208, 216

Buchheim, Hans 155, 192, $196 \mathrm{ff} ., 200$

Buchheim, Karl 194, $196 \mathrm{ff}$.

Bülow, Arthur 155

Caldera Rodríguez, Rafael 139,141

Calvani, Aristides 144

Câmara, Hélder Pessôa, gen. Dom Hélder 105, 166

Câmara, Jaime de Barros 91

Canaan, Gershon 133

Canaris, Wilhelm 68

Cantuti, Francesco 104

Carstens, Karl 162

Casaroli, Agostino 161

Castelli, Alberto 168,170

Castro Ruz, Fidel 136, 138, 171

Cicognani, Amleto Giovanni 166, 172 
Clay, Lucius D. 132, 134, 210

Colocci-Vespucci, Adriano Marchese 113

Conant, James 134

Cremer 62

Crivelli, G. 103

Czaja, Herbert 119, 176, 178f., 187

David, Eduard 42

Decker, Georg 45

De Gasperi, Alcide 115, 118

Deist, Heinrich 134

Delp, Alfred 55, 68, 95, 198, 204

Dessauer, Friedrich 35, 60, 211

Deuerlein, Ernst 193f., $196 \mathrm{ff}$.

Dibelius, Otto 179

Dietrich, Hermann Robert 94

Dietze, Constantin von 96

Dirks, Walter 177, 200

Dönhoff, Marion Gräfin 134

Döpfner, Julius $177 \mathrm{f} ., 183,187,199$

Dohrn, Klaus 90, 132, 134, 162, 196, 214

Dollfuß, Engelbert $36,71,90,112$

Don Sturzo, Luigi siehe Sturzo, Luigi

Dreyfus, Willy 75

Dulles, Allen W. 64, 211

Eberhard, Fritz (urspr. Helmut von Rauschenplat) 214

Ebert, Friedrich 155

Emmet, Christopher 132, 210

Erdmannsdörfer, Hans Gustav 49

Erhard, Ludwig 146, 148

Erkelenz, Anton 49

Erler, Fritz 14, 134, 148, $159 \mathrm{f}$.

Ersing, Joseph 60

Erzberger, Matthias 19

Eschenburg, Theodor 150

Eschmann, Ignatius 69

Esser, Thomas $54 \mathrm{f}$.

Etzel, Franz 69

Fahrenbrach, Heinrich 60

Falez, Stefan 105, 109

Falke, Gustav 70

Farny, Oskar 8, 62, 196

Faulhaber, Michael 83

Fedregotti, Graf Bossi 123

Fest, Joachim 178

Fischer, Ruth 209

Förster, Friedrich Wilhelm 84

Fonk, Wilhelm 62, 66, 194, $196 \mathrm{f}$.

Forschbach, Edmund $63 \mathrm{ff} ., 67 \mathrm{ff} ., 136$

Forster, Karl 147, 159, $190 \mathrm{f}$., $193 \mathrm{f} ., 196 \mathrm{ff}$.

Fortmann, Franz 47
Franken, Josef $33,136,155,196$

Franken, Paul 67ff., 194, 196

Freato, Sereno $141 \mathrm{f}$.

Freeden, Hermann von 71,73

Frei Montalva, Eduardo 141

Frey, Hans Günther 178

Frick, Wilhelm 59, 62, 192

Friedenthal, Richard 201

Friedrich, Carl Joachim 133, 207

Friedländer, Ernst 134, 212

Friedmann, Friedrich Georg 219

Frings, Joseph 99, 101 f., 110, 175, 187

Frisch, Alfred 201

Fritz, Gerhard 140

Froehling, Friedrich 103

Fütterer, Karl 69, 95, 99, 136, 152

Fugger von Glött, Joseph Ernst $197 \mathrm{f}$.

Gamper, Michael 112, 114, 117, 123

Gargitter, Josef 119, 126

Gaulle, Charles de 88, 90, 139, 182

Gauthier, Paul 166

Gawlina, Josef (Jozef Feliks) 110,187

Gayl, Wilhelm Freiherr von 27, 33

Gehlen, Reinhard 162

Geißler, Heiner 141

Gerbrandy, Pieter 90

Gerhardt, Stanislaw 142

Gerig, Otto 62

Gerstenmeier, Eugen 102

Gewandt, Heinrich 140f., 144

Gibbert, Paul 8

Gibson, Hugh Simons 106

Gisevius, Hans Bernd 64

Globke, Hans 143, 145, 149, 153, 162

Glorieux, Achille 168

Goebbels, Joseph 66

Goerdeler, Carl 69, 97

Goergen, Hermann Mathias $84 \mathrm{f} ., 140 \mathrm{f}$.

Göring, Hermann 55

Goldie, Rosemary 168

Gomulka, Wladyslaw 181

Gordan, Paulus 81, 88, 104, 108, $179 \mathrm{f}$.

Goulart, João 142

Graf, Otto 64

Graham, Robert 123

Grégoire, Pierre 201

Gregor XVI. 184

Gremillion, Joseph 170

Gröber, Konrad 62

Grollmus, Maria 38 f., 70

Groß, Nikolaus 55, 68f., 95

Gruber, Karl 115

Guidotti, Gastone 119 
Gundlach, Gustav 102, 159, $197 \mathrm{f}$.

Gurian, Waldemar 38, 70, 87, 200, 206, 208

Gurion, David Ben 139

Guttenberg, Karl Theodor Freiherr von und zu 14, $134 \mathrm{f} ., 145-150,156,159,162,176$, $179,198,209$

Habermann, Max 69

Hackelsberger, Albert 59,61f.

Haefs, Johann H. 99, 109

Häntzschel, Kurt 82

Hahn, Georg 75

Hahn, Karl Josef 140f., 220

Haltmayer, Josef 101, 109

Hamacher, Wilhelm 69

Hammerstein-Equord, Kurt Frhr. von 69

Hans, Werner 45

Hanssler, Bernhard $196 \mathrm{ff}$.

Haubach, Theodor 50

Heck, Bruno 138, 140f., 145, 149f., 158

Heiden, Konrad 36

Heile, Wilhelm 49

Heinemann, Rolf 83

Heinen, Heinrich 97

Helble, Max 99

Heller, Hermann $37 \mathrm{f}$,

Henckel von Donnersmarck, Hans Graf 75

Hengsbach, Franz 194

Henrich, Franz 199

Hensel, Walther 67ff., 96, 205

Herions, Josef 81

Herkenrath, Adolf 142, 144

Hermens, Ferdinand A. 45, 48, 89, 147, 151,206

Hermes, Andreas 50, 97, 152, 190, $194 \mathrm{f}$, 215

Herter, Christian 134

Herwarth von Bittenfeld, Hans 153

Hesslein Pablo (Paul) 87

Heuss, Theodor 40, 49, 96, 135, 152, 197

Hildebrand, Dietrich von 90,200

Himmler, Heinrich 63,113

Hindenburg, Oskar von 66

Hindenburg, Paul von 33, 63, 65

Hirsch, Felix R. 133

Hirschfeld 75

Hirschmann, Johannes (Hans, Johann Baptist) 194, 196

Hitler, Adolf $11,54,58-61,63-67,69,78$, $87,89,112,192,205$

Hlond, August 174

Höfer, Josef 198

Hoegner, Wilhelm 132, 214

Hofer, Peter $113 \mathrm{f}$.
Hoffmann, Johannes 11, $84 \mathrm{ff} ., 200$

Hoffmann-Harnisch, Wolfgang 96

Holborn, Hajo 132f., 213

Horkheimer, Max 201

Hugenberg, Alfred 53, $58 \mathrm{f}$.

Imbusch, Heinrich 60

Ingrim, Robert 208

Isay, Rudolf 80,82

Jacobsen de Rosen, Pierre 106

Jaeger, Hans 201

Jaeger, Richard 135

Jaksch, Wenzel 119,134

Jedin, Hubert $178,197 \mathrm{f}$.

Johannes XXIII. (Angelo Giuseppe Roncalli) 165

Johannes Paul II. (Karol Wojtyla) 179, 181, 183

John, Dr. 51

Joos, Joseph 35, 60f., 68, 196

Jung, Edgar Julius 64-68

Jung, Carl Gustav 204

Kaas, Ludwig 52, 54-62, 77, 87, 91, $93 \mathrm{f}$., $99,102,125,129,184,189-194$

Kadow, Manès 69

Kafka, Gustav E. 159

Kahr, Gustav Ritter von 67

Kaisenberg, Georg 48

Kaiser, Hermann George 133

Kaiser, Jakob 8, 60, 68f.

Kann, Friedrich 30

Kannengießer, Josef 196

Kaphan, Heinrich 82

Kaskell, Joseph 133

Katz, Rudolf 208

Kellen, Stephen 133

Keller, Thomas 88

Kempf, Nikolaus 81

Keudell, Walter von 46

Keup, Erich 25

Kiesinger, Kurt Georg 8, 135, 145, 148$151,156 \mathrm{f} ., 160,162,181$

Killion, Edward G. $103 \mathrm{f}$.

Kindt-Kiefer, Jakob 94, $131 \mathrm{f} ., 211$

Kirchfeld, Franz 75

Kissinger, Henry A. 134, 215

Klaiber, Manfred 14, 119

Klausener, Erich 67

Klemperer, Alfred von 134

Klepper, Otto 155

Klepsch, Egon 141

Kluge, Hans von 66 
Kluthe, Hans Albert 201

Knab, Otto Michael 38

Knoeringen, Waldemar von 10, 159, 209

Koch-Weser, Erich 11, 42, 46, 71-74, $81 \mathrm{f}$., 88f., 98, 148

Koch-Weser, Geert 30, 73, $82 \mathrm{f}$.

Koch-Weser, Volker 148

Koeppler, Heinz 209

Körner, Heinrich $68 \mathrm{f}$.

Koestler, Arthur 208

Kogon, Eugen 91

Kohn-Brandenburg, Alexander 159

Kolb, Walter 36

Kominek, Boleslaw 173, 176-179, 181-184, 187

Kopecky, Jaromir 84

Kopf, Hermann 14, 35 f., 75, 95, 102, 119 , $135 \mathrm{f} ., 148$

Kraemer, Fritz 215

Kramer, Franz Albert $94 \mathrm{f}$., $211 \mathrm{ff}$.

Kraske, Konrad 140

Krausnick, Helmut 12

Kreiser, Walter 85

Krone, Heinrich 8, 14, 21, 40, 46, 51, 62, 96, $118 f$., 136, $142 \mathrm{f}$., $145 \mathrm{ff} ., 149,153,155$, 157, 162, 190 f., 193 f., 196 f., 215

Krüger, Walter 155

Kühr, Fritz (Friedrich) 36, 81 f., 90

Külz, Wilhelm 46, 49

Kunst, Hermann 158

Lanctot, John 104, 108

Landauer, Carl 133

Landi, Andrea P. 105

Landi, Andrew 103

Langemann, Hans 163

Langer, Alexander 126

Langer, Arthur 126

Langer, Lilly 126

Lanz, Renate 76

Lasky, Melvin 214

Leber, Georg 144, 147, 156, 160 f., 182

Lehmann, Reinhold 179

Lehr, Robert 69

Leiber, Robert 98 f., 101 f., 104, 109 f., 127, 190,193 f., 196

Lemke, Helmut 158

Lenné, Albert 62

Lenz, Carl Otto 135

Lenz, Heinrich 83

Leo XIII. (Vicenzo Gioacchini Pecci) 17

Lercaro, Giacomo 166

Leone, Giovanni 123

Letterhaus, Bernhard 55, 61, 68, 95, 215
Leuschner, Wilhelm 69, 205

Lichteritz 36

Ligutti, Luigi 94, 101-103, 170

Lochner, Louis P. 210

Löwenstein-Wertheim-Freudenberg, Hubertus Prinz zu 210

Lovat, Lord 72

Luce, Claire Boothe 191

Luce, Henry 133

Lübke, Friedrich Wilhelm $71 \mathrm{ff}$.

Lübke, Heinrich $14,51,71,99,102,109$, 146f., 149, 151-159, 162

Lücke, Paul 14, 146-150

Lukaschek, Hans 101

Luther, Hans $71 \mathrm{f}$.

Macharski, Franciszek 188

Machui, Artur von 30

Mager, Agnes 70, 75

Mager, Hermann 83

Mager, Johannes 83

Magnago, Silvius 123

Maier, Max Hermann 82f., 96

Maier-Hultschin, Johannes 200

Majonica, Ernst $175 \mathrm{f}$.

Man, Hendrik de 37

Mann, Golo 201

Mann, Thomas 88

Marckwald, Joachim 82

Mariaux, Franz 66

Marx, Wilhelm 35

Maßmann, Karl 30

Mattick, Kurt 135

McGeough, Joseph 103

McCloy, John 132

Mehnert, Klaus 135

Melchers, Paulus 18

Mende, Erich 135

Metzger, Max Josef 180

Mierendorff, Carlo 45, 47, 49f., 89

Mikat, Paul 196, $197 \mathrm{f}$.

Möller, Hans 135

Möller, Horst 12

Molt, Peter 138-142

Moltke, Helmuth Graf von 198, 204

Mommer, Karl 134

Montini, Giovanni Battista siehe Paul VI.

Montini, Ludovico 159

Morgenthau, Hans Joachim 205, 207

Morgenthau, Henry $132 \mathrm{f}$.

Morsey, Rudolf 7, 189-200, 216

Moser, Edmund 144

Moser, Gerold 144

Mota de Vasconcelos, Carlos Carmelo 91 
Muckermann, Friedrich 66, 200

Muehlen, Norbert $132 \mathrm{f}$.

Müller, Artur 71

Mueller, Franz H. 35

Müller, Hermann 40

Müller, Josef 68

Müller, Karl 152

Müller, Otto 68

Muench, Aloysius 195

Murri, Romolo 112

Mussolini, Benito 76, 78, 112

Nahm, Peter Paul 179

Nannen, Henri 154

Naumann, Friedrich 42, 49

Nell-Breuning, Oswald von $28,38,101$, 166, 170

Nellen, Peter 159

Neumark, Fritz 95, 211

Neurath, Konstantin Freiherr von 33

Neuß, Wilhelm 189

Nevermann, Paul 50

Niebuhr, Reinhold 38, 209

Niemöller, Martin 176

Niklas, Wilhelm 152

Nixdorf, Oswald $72 \mathrm{f}$.

Norris, James J. 94, 102 ff., 170

Nuschke, Otto 49

Oberländer, Theodor 182

O'Grady, John 103

Ollenhauer, Erich 146

Oppenheimer, Franz M. 133

Oppenheimer, Fritz E. 133

Ossietzky, Carl von 85

Pacelli, Eugenio siehe Pius XII.

Pachter, Henry M. 133

Pahl, Walter 50

Pant, Eduard 200

Papen, Franz von 34, 55, 58, 64-67

Paul VI. (Giovanni Battista Montini) 101, 103f., 159f., 161, 165, 182, 191

Paul, Ernst 159

Pawliczek, Josef 179

Pecci, Vicenzo Gioacchini siehe Leo XIII.

Pechel, Rudolf 64, 69

Perlitius, Ludwig 54f., 58, 192, 195

Pesch, Heinrich 140

Peters, Hans $197 \mathrm{f}$.

Pfister, Karl 49

Pieper, Josef 135

Pius IX. (Graf Giovanni Mastei-Ferretti) 165
Pius XI. (Achille Ratti) 38

Pius XII. (Eugenio) 77, 83, $93 \mathrm{f.,} 100 \mathrm{ff}$., $127,189,194$

Platz, Hermann 36

Possony, Stefan Thomas $206 \mathrm{ff}$.

Preuß, Helmuth 35

Prinz, Joachim 134

Probst, Adalbert 67

Ranke, Eugen 83

Rathenau, Walter 35

Ratti, Achille siehe Pius XI.

Rauschning, Hermann 38

Reichenau, Walter von 66

Reichmann, Eva 201

Reichwein, Adolf 50

Renger, Annemarie 160

Repgen, Konrad 196ff., 200

Ritzel, Heinrich 59

Roegele, Otto B. $197 \mathrm{f} ., 211$

Röpke, Wilhelm 94f., 211, $213 \mathrm{f}$.

Romano, Antonio 118

Roncalli, Giuseppe Angelo siehe Johannes XIII.

Rosenberg, Ludwig 214

Rothfels, Hans 206

Roy, Maurice 166, 168, 170

Rubin, Ladislao 179

Rüstow, Alexander 95, 211

Ruge, Gerd 135

Ruhnau, Heinz 135

Ruiz-Gimérez, Joaquín 143

Rumor, Mariano 118, 142

Rundstedt, Gert von 66

Sangnier, Marc 36,180

Sattler, Dieter 14, 135f., 158, 197

Seeckt, Hans von 173

Seelos, Gebhard 142

Seger, Gerhart $208 \mathrm{f}$.

Segni, Antonio 155

Sehrbrock, Hermann 153

Sering, Max $25 \mathrm{f}$.

Shuster, George N. 200, 206, $209 \mathrm{f}$.

Siegmund-Schultze, Friedrich 94

Simmel, Oskar 192

Simon, Hugo $84 \mathrm{f}$., 87

Simons, Hans $38,50,83$

Sinzheimer, Hugo 50

Sinzig, Petrus 87

Sirutis, Stany 105

Smolka, Georg 189f., 194, $196 \mathrm{f}$., 200

Sollmann, Wilhelm 208 
Sonnenschein, Carl 7, 20f., 36, 47, 76, 112, 148,158

Spaak, Paul Henri 90

Spahn, Martin 64

Sperr, Franz 198

Spiecker, Carl 97

Spindler, Max 198

Splett, Carl Maria 176

Suenens, Leo Jozef 166

Süsterhenn, Adolf 192, 211

Swanstrom, Edward E., 103

Schäfer, Johann 99

Schaefer, Karl Heinz $121 \mathrm{f}$.

Schäffer, Fritz 198

Scharmitzel, Theodor 69

Scharnagl, Anton 192

Scharp, Heinrich 196

Scheel, Walter 138

Scherpenberg, Albert Hilger van 159

Schiller, Karl 135

Schlamm, William S. $207 \mathrm{f}$.

Schlange-Schöningen, Hans $32 \mathrm{ff} ., 52$, $71 \mathrm{ff} ., 98,152$

Schleicher, Kurt von $52 \mathrm{ff}$., $66 \mathrm{f}$.

Schlüter-Hermkes, Maria 135

Schmaltz, Kurt 19

Schmid, Carlo 135, 159, 175

Schmidt, Helmut 135, 158

Schmitt, Hermann Josef 55, 60, 64f., 68, 91, $95,190,194,196$

Schmitt-Vockenhausen, Hermann 135

Schnabel, Franz 189

Schreiber, Georg $190 \mathrm{ff}$.

Schröder, Paul 160

Schürholz, Franz 155

Schulenburg, Fritz-Dietlof von der 204

Schulte, Karl Joseph 62

Schumacher, Kurt 98, 213

Schuman, Robert 109, 211

Schuschnigg, Kurt von 82

Schwarz, Albert $196 \mathrm{f}$.

Schwarz, Hans-Peter 212, 214

Schwarzschild, Leopold 133, 207

Schwend, Karl 194, 196

Schwerin, Friedrich von 25, 27

Schwering, Leo 196

Stadtler, Eduard 64, 68

Stalin, Josif Wissarionowitsch $11,90,112$

Stammer, Otto 35

Stampfer, Friedrich 192, 208

Stark, Tadeusz 110

Stasiewski, Bernhard 190, $196 \mathrm{ff}$.

Staudinger, Hans $132 \mathrm{f}$.
Steger 75

Stegerwald, Adam $31 \mathrm{ff}, 197$

Stehle, Hansjakob 178, 187

Steinhoff, Johannes 135

Steinmann, Prälat 125

Sternberger, Dolf 147

Stinnes, Edmund 75

Stolper, Gustav $207 \mathrm{f}$.

Stoltenberg, Gerhard 140

Stomma, Stanislaw 176

Straßer, Gregor 67

Strauß, Franz Josef 146

Strauss, Leo $206 \mathrm{f}$.

Studenic, Hubert (Pseudonym siehe Simon, Hugo)

Sturzo, Luigi (eigentl. Luigi Boscarelli, gen. Don Sturzo) 112,118

Tecklenburg, Adolf 45

Teipel, Heinrich $45 \mathrm{ff}$.

Tenhumberg, Heinrich 160, 179, 187

Teusch, Christine 60, $194 \mathrm{f}$.

Teusch, Josef 179

Thaler, Andreas $71 \mathrm{ff}$.

Thieme, Karl Otto 14, 38 f., 68 f., 88, 90, $94 \mathrm{f} ., 99,133,189 \mathrm{f} ., 198$

Thoma, Richard 45

Thomas, Arthur H. M. 72

Thompson, Dorothy 210

Tillich, Paul 37f., 209

Tinzl, Karl 114, 118f.

Treviranus, Gottfried Reinhold 11, 87, 97, 197,210

Tolomei, Ettore 113

Torella y Cascante, Ramón 170

Tschurtschenthaler 126

Turowicz, Jerzy $176 \mathrm{f}$.

Ulitzka, Carl 59

Ulich-Beil, Else 45

Unkelbach, Helmut 151

Unterrichter, Rudolf Frhr. von 119f., 126

Unterrichter, Christoph Frhr. von 105, 107

Vageler, Paul 71

Vanistendael, August 139-142, 168, 170

Vansittart, Robert 133

Vargas, Getúlio 83, 85

Vargas, Benjamino 86

Veronese, Vittorino 168

Villot, Jean Marie 172

Vicentini, Giovanni Battista 103, 105

Vockel, Heinrich $21,40,45,47,51,62,190$, 194 
Voegelin, Eric 206

Vogel, Bernhard 141

Waldmann, Eric 133

Wallenberg, Hans 134

Wallich, Henry 133

Warburg, Eric 75, 132

Weber, Alfred 211

Weber, Helene 8,60f., 135, 196

Weber, Julius 50

Weber, Max 49

Wegmann, August 61, 196

Wehner, Greta 14, 157

Wehner, Herbert $8,10,14,39,146-151$, $153 \mathrm{f} ., 156-161,163,178 \mathrm{f} ., 181 \mathrm{f}$.

Weizsäcker, Richard von 182

Wenger, Paul Wilhelm 211

Wesoly, Szczepan 179

Wessel, Gerhard 162

Westrick, Ludger 141

Winowska, Maria 179
Wirmer, Josef $68 \mathrm{f}$.

Wirth, Joseph 14, $34 \mathrm{f} ., 38,45,48,51,58$, $60 f ., 63,76,82,90,94$ f., 98, 132, 173, 211

Wissing, Wilhelm 198

Wittfogel, Karl 208

Witzleben, Erwin von 66

Wohlrabe, Jürgen 141

Wolfers, Arnold 134, 199

Wolff, Karl 123

Wolff von Amerongen, Otto 135

Wolker, Ludwig 29, 67, 70

Wojtyla, Karol siehe Johannes Paul II.

Wosnitzer, Franz 109

Württemberg, Odo von 84

Wüstenberg, Bruno 157, 159, 194

Wyszynski, Stefan 176f., 179-183, 187

Zeegers, G. H. 111

Zingerle, Josef 118

Zittel, Bernhard $197 \mathrm{f}$.

Zweig, Stefan 86 